\title{
Controlling the Packing of Metal-Organic Layers by Inclusion of Polymer Guests
}

Benjamin Le Ouay, ${ }^{\dagger}$ Hikaru Takaya, ${ }^{\ddagger}$ Takashi Uemura ${ }^{*}$,, ,\#

${ }^{\dagger}$ Department of Advanced Materials Science, Graduate School of Frontier Sciences, The University of Tokyo, 5-1-5 Kashiwanoha, Kashiwa, Chiba 277-8561, Japan.

‡ Institute of Chemical Research, Kyoto University, Gokashou, Uji, Kyoto 611-0011, Japan

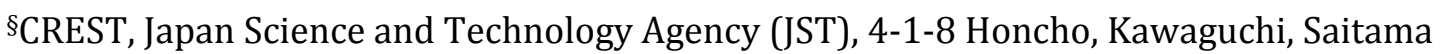
332-0012, Japan.

\#Department of Applied Chemistry, Graduate School of Engineering, The University of Tokyo, 7-3-1 Hongo, Bunkyo-ku, Tokyo 113-8656, Japan.

*t-uemura@k.u-tokyo.ac.jp 


\section{Materials and methods:}

Before use, azobisisobutyronitrile (AIBN) was purified by recrystallization from ethanol, and styrene was purified by distillation under vacuum. Other reagents were used as received.

Synthesis of $\left[\mathrm{Zn}_{2}(\mathrm{BDC})_{2}(\mathrm{DABCO})\right]_{\underline{\underline{n}}} \underline{(\mathbf{1})}$

1 was prepared according to literature. ${ }^{1} 2.00 \mathrm{~g} \mathrm{Zn}\left(\mathrm{NO}_{3}\right)_{2} \bullet 6 \mathrm{H}_{2} \mathrm{O}, 1.12 \mathrm{~g}$ terephthalic acid $\left(\mathrm{H}_{2} \mathrm{BDC}\right)$, $0.37 \mathrm{~g}$ 1,4-Diazabicyclo[2.2.2] octane (DABCO) and $80 \mathrm{ml}$ of DMF were introduced in a screwcap vial, and stirred until a homogeneous white suspension was obtained. The reaction vessel was then placed at $120^{\circ} \mathrm{C}$ for $48 \mathrm{~h}$. After cooling, the MOF (white powder) was collected by filtration, and further purified by stirring in anhydrous DMF for $2 \mathrm{~h}$ at $80^{\circ} \mathrm{C}$. The MOF was then collected by filtration and further washed with anhydrous DMF and methanol. Solvent was then removed overnight at $150{ }^{\circ} \mathrm{C}$ under vacuum, and samples were stored activated, over desiccating silica.

\section{$\underline{\text { Synthesis of } 1 \supset \mathrm{PSt}}$}

Activated 1 (ca. $500 \mathrm{mg}$ ) was introduced in a round-bottom flask closed with a 3-ways tap, and put under $\mathrm{N}_{2}$ atmosphere. In a vial, AIBN ( $35 \mathrm{mg}$ per $1 \mathrm{~g}$ of MOF) was dissolved in freshly distilled styrene (ca. $3 \mathrm{ml}$ ). The styrene was then introduced in the MOF flask through the 3-ways tap, under $\mathrm{N}_{2}$ flow, so that the MOF was entirely covered by liquid. Excess styrene was then removed under reduced pressure $(0.3 \mathrm{kPa})$, until the powder was dry. A mass increase of $520 \mathrm{mg}$ per $1 \mathrm{~g}$ of MOF corresponded to 1 fully loaded with styrene, without monomer remaining outside of the MOF. The flask was then filled with $\mathrm{N}_{2}$, and maintained at $60{ }^{\circ} \mathrm{C}$ for a given period of time. Styrene conversion could be controlled by adjusting the reaction time (see Figure S29). Unless specified, $1 \supset$ PSt refers to the sample with a styrene conversion of $52 \%$. After reaction, composites were washed with anhydrous $\mathrm{MeOH}$, and activated at $150{ }^{\circ} \mathrm{C}$ under vacuum. Samples were stored activated over desiccating silica.

\section{$\underline{\text { Heat-treatment of } 1 \text { and } \mathbf{1} \supset \mathrm{PSt}}$}

Samples (ca. $250 \mathrm{mg}$ ) were placed in an alumina crucible and introduced in a quartz tubular furnace. The tube was put under vacuum, then heated at $320^{\circ} \mathrm{C}$ for $2 \mathrm{~h}$. A cold trap was set before the pump, to collect sublimated DABCO. After cooling down, samples were stored over desiccating silica.

\section{Instrumentation}

PXRD diagrams were collected on a Rigaku SmartLab Diffractometer, using a $\mathrm{Cu}$ anode and a $\mathrm{K}_{\alpha}$ monochromator $(\lambda=0.154 \mathrm{~nm})$. Variable temperature PXRD measurements were performed under a $\mathrm{N}_{2}$ flux, using the built-in DSC module. $\mathrm{N}_{2}$ adsorption measurements were performed on a BELSORP II mini (BEL-Japan, Inc.). Heptane adsorption measurements were performed on a BELSorp Aqua 3 (BEL-Japan,Inc.). Samples were activated at $120{ }^{\circ} \mathrm{C}$ for $16 \mathrm{~h}$ before the measurement. Differential scanning calorimetry plots were collected on a DSC7020 Thermal Analysis System (Hitachi). Measurements were performed in an Al closed pan, under a $\mathrm{N}_{2}$ flux. Thermo-gravimetric analysis (TGA) plots were recorded using a Rigaku TG 8120. Gel permeation chromatography (GPC) measurements on PSt were performed in chloroform at $40^{\circ} \mathrm{C}$ on three linear-type polystyrene gel columns (Shodex K-805L) that were connected to a JASCO PU-980 precision pump, a JASCO RI-930 refractive index detector, and a JASCO UV-970 $\mathrm{UV} /$ vis detector set at $256 \mathrm{~nm}$. The columns were calibrated against standard polystyrene samples. 


\section{XAFS measurement}

Zn K-edge XANES and EXAFS measurements were performed at the BL14B2 beamline of the synchrotron SPring-8 in Japan under standard beamline conditions. The Zn K-edge $(9.67 \mathrm{keV})$ XAS data were corrected by transmission mode using $\mathrm{N}_{2} / \mathrm{Ar}$ mixed gas-filled ionization chambers with optimized gas ratio and pressure with $\mathrm{Si}$ (111) double-crystal monochromator. For an energy calibration of XANES spectra of Zn K-edge, a zinc metal foil was used as reference sample to set the inflection point determined by the maximum first-derivative of XANES spectra to $9659.095291 \mathrm{eV}$. For solid-state XAFS, BN pellets (ca. $0.5 \mathrm{~mm}$ thickness) were prepared by mixing of the powders of MOF and a commercial BN powder for dilution, and then uniaxiallycompressing by tableting machine. All the processes for sample preparation were performed in an argon-filled glovebox. XAFS data processing and EXAFS fitting simulation were carried out by using Athena and Artemis software through the embedded FEFF6 program. ${ }^{2}$

O K-edge NEXAFS measurement was performed at the C-branch of BL27SU beamline of SPring$8 .^{3}$ The MOF powder samples were fixed with conductive double-sided carbon tape onto a cupper sample holder. The sample holder was fixed on a linear and rotatable manipulator and installed into a vacuum chamber which was evacuated to a pressure of less than $1 \times 10^{-5} \mathrm{~Pa}$. The $\mathrm{O} \mathrm{K}$-edge NEXASF spectra (500-570 eV) were taken in FY spectra using a silicon drift detector (SDD). The SDD was mounted perpendicularly to the incident photon beam axis. The monochromatic light was irradiated at an angle of about $80^{\circ}$ relative to the sample normal to minimize the contamination of the elastic scattering.

$\mathrm{N}$ K-edge NEXAFS measurement was performed at the soft X-ray beamline BL3U at the UVSOR-III Synchrotron. ${ }^{4}$ The MOF powder samples were fixed with conductive double-sided carbon tape onto a stainless sample holder. The sample holder was fixed on a rotatable linear and installed into a vacuum chamber which was evacuated to a pressure of less than $1 \times 10^{-5} \mathrm{~Pa}$. The N K-edge NEXASF spectra $(400-430 \mathrm{eV})$ were taken in total electron yields (TEYs) by measuring a sample drain current. The energy resolution of the incident soft X-rays at the N Kedge is set to $0.2 \mathrm{eV}$. Subtraction of background of carbon tape, baseline correction, and normalization of the resulting spectra were performed by using Athena program.

\section{FEFF fitting analysis of $1,1 \supset$ PSt, $1 \mathrm{HT}$, and $1 \mathrm{HT} \supset$ PSt.}

For FEFF fitting analysis of DABCO-pillared MOF of 1 (Figures S13-S16) and $1 \supset$ PSt (Figures S17-S20), the atomic coordinates obtained from single crystal X-ray structure of 1 (Figure S10, Table S1) and DFT-based optimized structure of 1 (Figure S11, Table S2) were used. For the DFT-based geometry optimization of $\mathbf{1}$, the molecular geometry and the position of disordering DABCO pillar ligand were optimized with fixing the initial lattice parameter of single crystal X-ray structure of $\mathbf{1}$. For FEFF fitting analysis of $1 \mathrm{HT}$ and 1HTつPSt, a DFT-based non-pillared structure was prepared and used for the fitting model (Figure S12, Table S3). The DFT calculation was performed by using CASTEP $^{5}$ code embedded in BIOVIA Material Studio 2018 with GGA/RPBE under the on-the-fly generated ultrasoft pseudopotential. The cell- and molecular geometryoptimization were simultaneously performed under a periodic boundary condition by using the atomic coordinate and lattice parameter derived from CIF data of $\mathbf{1}$ as the initial cell and molecular structure. For comparison of all the above molecular geometries, the atomic distances and bond angles of MOF in the crystal phase, DFT-optimized DABCOpillared geometry, DFT-calculated non-DABCO-pillared geometry were summarized in Table S1. 


\section{Simulation of $O$ K-edge NEXAFS spectrum of 1 and 1 HT.}

Simulation of O K-edge NEXAFS spectra of $\mathbf{1}$ and non-DABCO-pillared MOF of 1HT were performed by code embedded in BIOVIA Material Studio 2018 with GGA/RPBE under the on-the-fly generated ultrasoft pseudopotential. ${ }^{6}$ The core level excitation process was simulated including an appropriate core-hole effect based on the optimized structure shown in Figure S11 and S12 for $\mathbf{1}$ and $\mathbf{1 H T}$, respectively. 
A

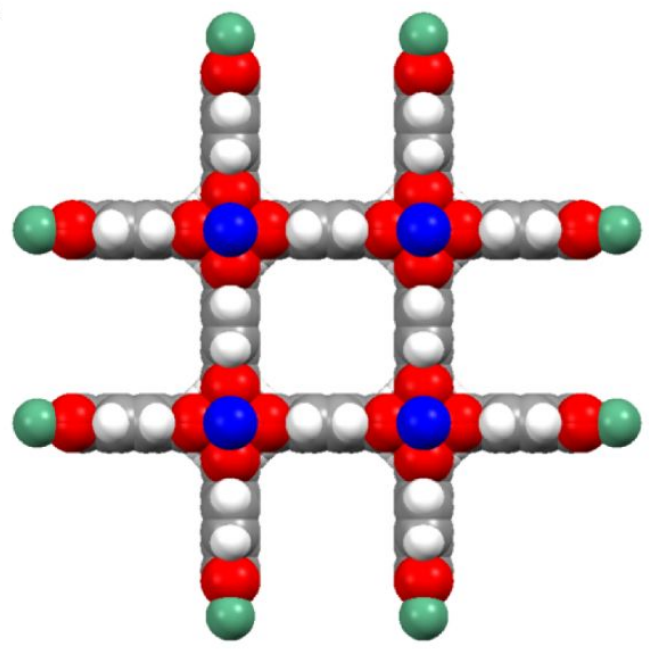

B

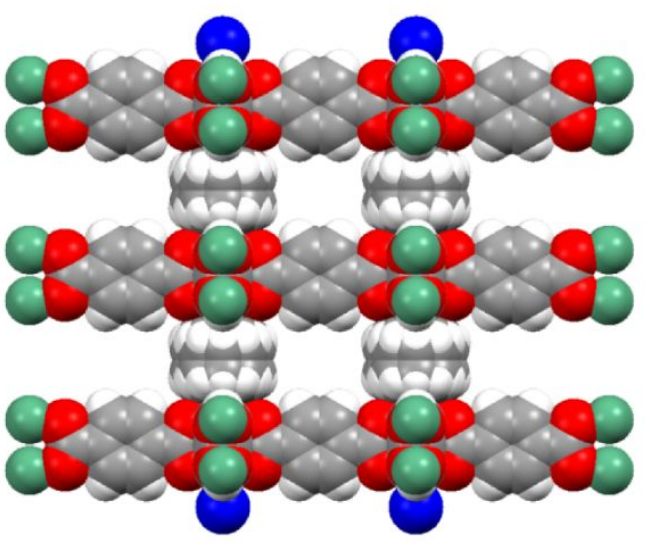

Figure S1. Crystal structure of 1, as described in $\operatorname{Ref}^{1}{ }^{1}$ (A) View along the nanochannels ( $c$-axis). The window dimensions along this axis are $7.5 \times 7.5 \AA^{2}$. (B) View perpendicular to the nanochannels, along the BDC ligands ( $a$-axis). The window dimensions along this axis are $5.0 \times$ $3.5 \AA^{2}$. The view along the $b$-axis is equivalent. (Grey: carbon; Red: oxygen; White: hydrogen; Blue: nitrogen; Green: zinc). Given the size of the styrene monomer $\left(6.8 \times 4.4 \AA^{2}\right)$, polymerization proceeds only along the $c$-axis nanochannels. The PSt chain alignment along the $c$-axis has also been confirmed experimentally in analogous frameworks. ${ }^{7}$

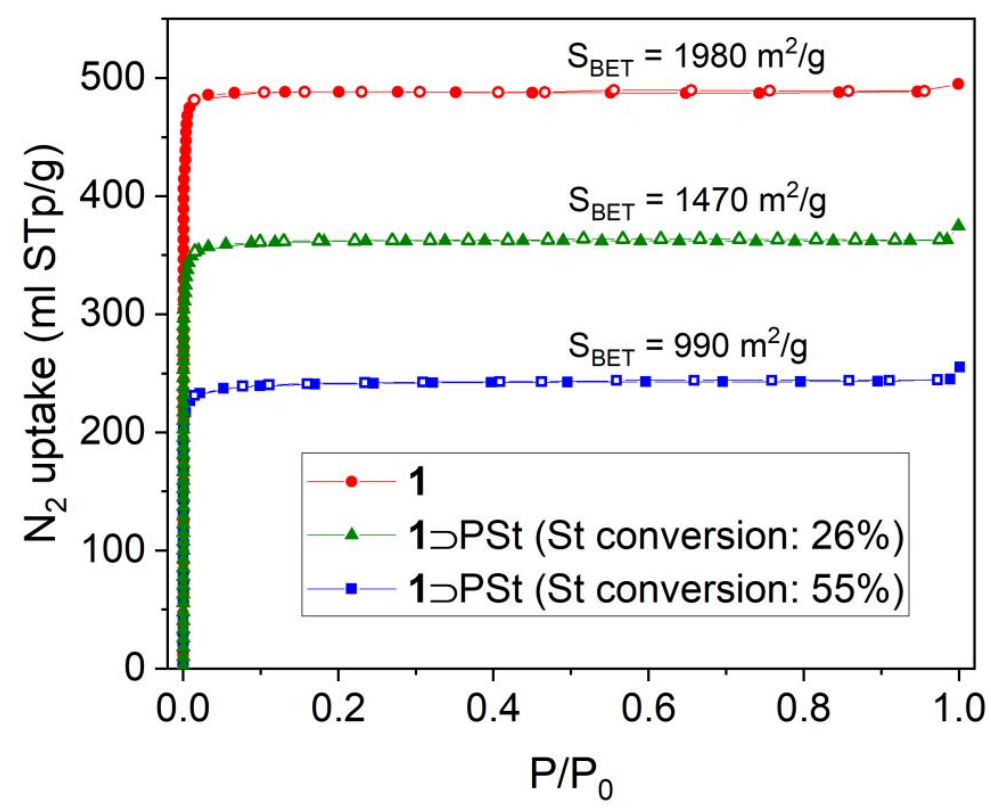

Figure S2. $\mathrm{N}_{2}$ adsorption isotherms (77 K) of $\mathbf{1}$ and $\mathbf{1} \supset \mathrm{PSt}$ with various St conversions. 


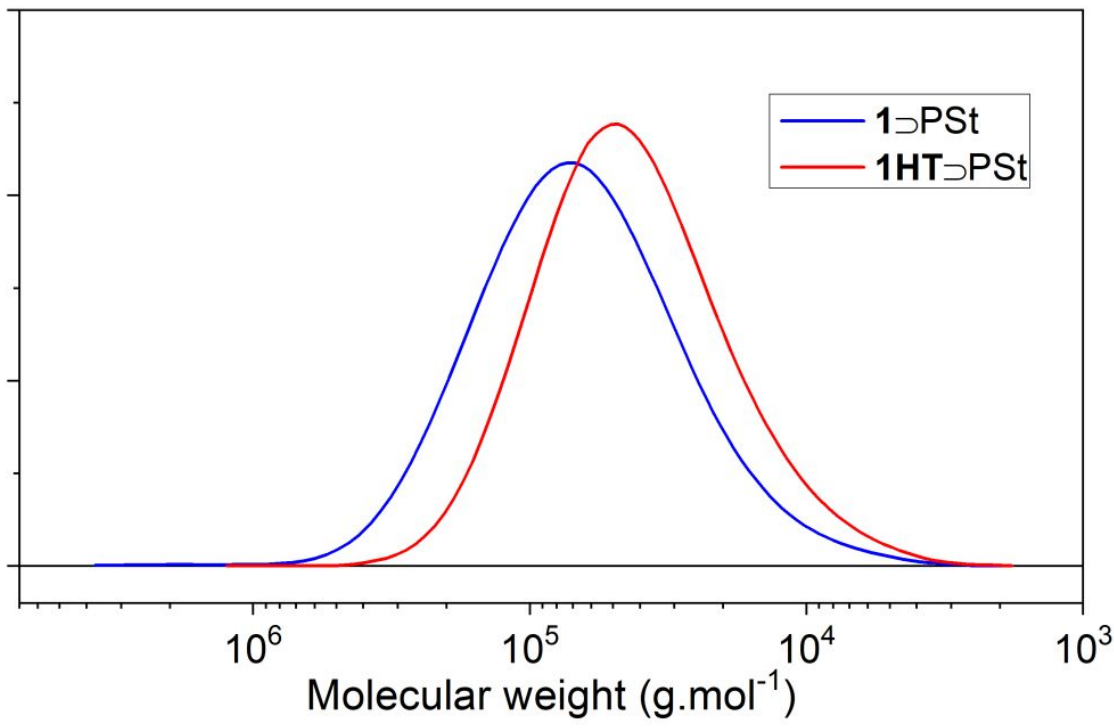

Figure S3. Molecular weight distribution of PSt extracted from $1 \supset$ PSt $\left(M_{\mathrm{n}}=45,000, \oslash=2.1\right)$ and 1HT $\supset$ PSt $\left(M_{\mathrm{n}}=31,000, D=1.9\right)$, obtained by GPC. While some chain shortening may happen during the heat-treatment, the molecular weight of PSt remains high, allowing a threading through multiple layers. 

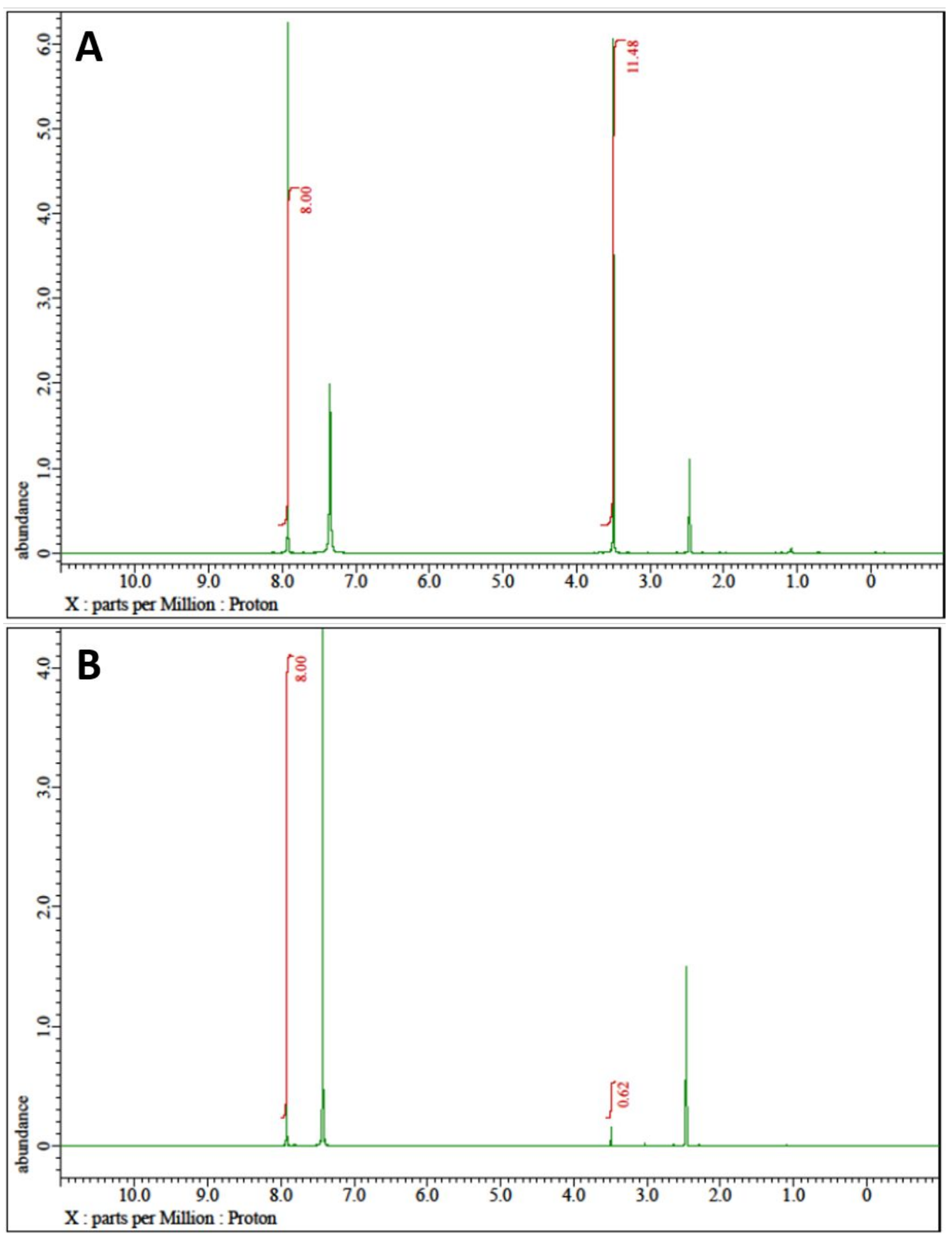

Figure S4. Continued. 


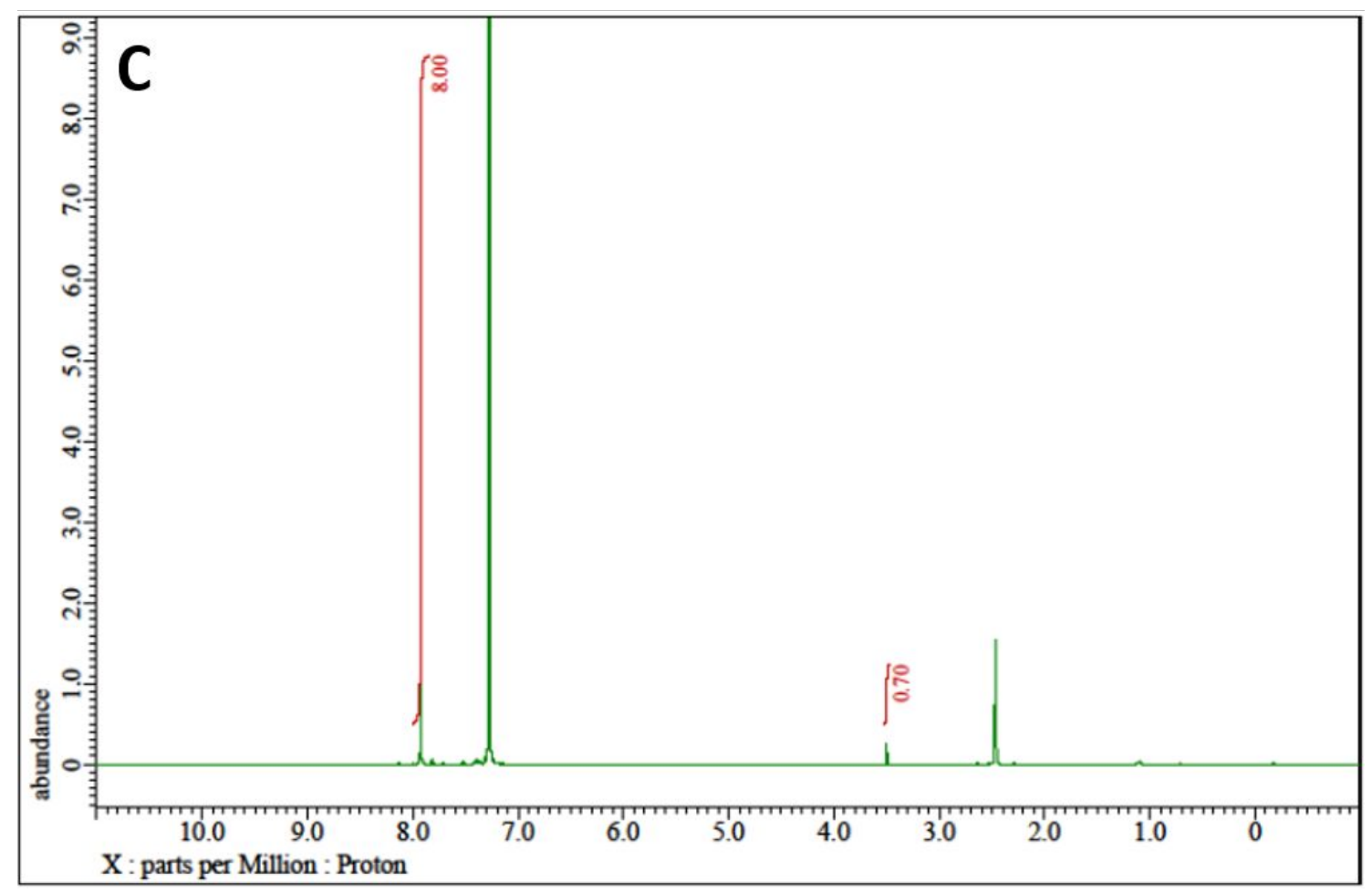

Figure S4. NMR spectra of 1 (formula: $\left(\mathrm{Zn}_{2}(\mathrm{BDC})_{2}(\mathrm{DABCO})\right)(\mathbf{A}), \mathbf{1 H T}(\mathbf{B})$ and $\mathbf{1 H T} \supset \mathrm{PSt}(\mathbf{C})$. The peak of $\mathrm{H}_{2} \mathrm{BDC}$ (singlet, $7.92 \mathrm{ppm}$ ) was normalized as 8 . The DABCO content was determined from the integral of the corresponding peak (singlet, $3.50 \mathrm{ppm}$ ). The theoretical value of this integral in $\mathbf{1}$ is 12 . Note that PSt precipitated after decomposition of the host, and was removed by filtration. Spectra were measured in a DMSO- $\mathrm{d}_{6} / \mathrm{DCl}\left(35 \mathrm{w} \%\right.$ in $\left.\mathrm{D}_{2} \mathrm{O}\right)$ mixture $(9: 1$ v:v). Solvent peaks: $2.50 \mathrm{ppm}$ (DMSO methyl) and 7.25-7.45 ppm (acidic protons). 


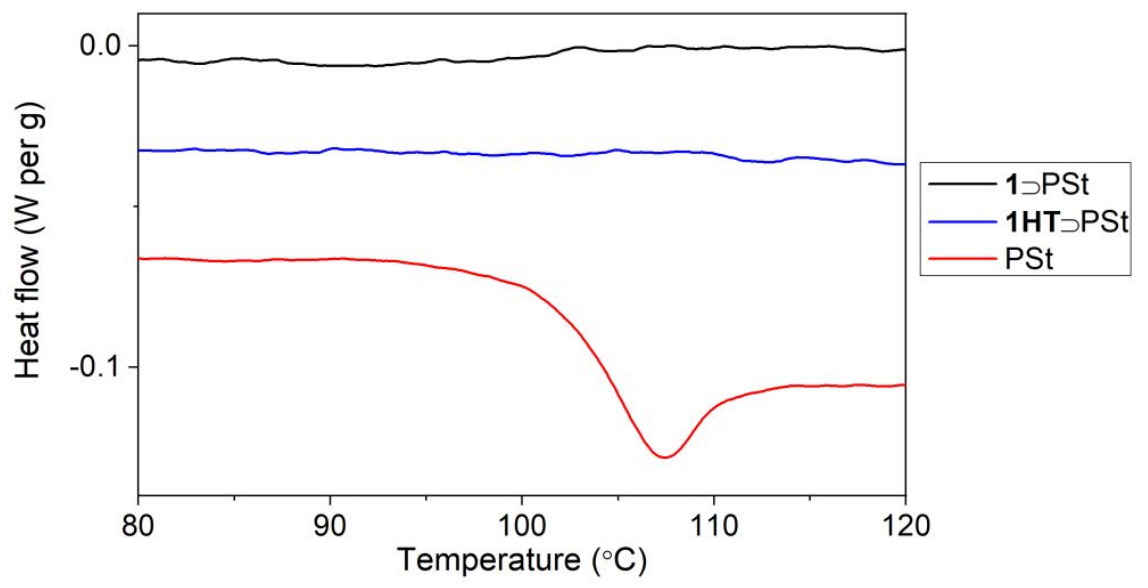

Figure S5. DSC analysis of $1 \supset$ PSt, 1HTدPSt and PSt extracted from 1HTכPSt. While a glass transition was clearly visible at $103{ }^{\circ} \mathrm{C}$ for free PSt, no such behavior was observed for the composites, due to the sequestration of chains in the MOF nanochannels. ${ }^{8}$ 


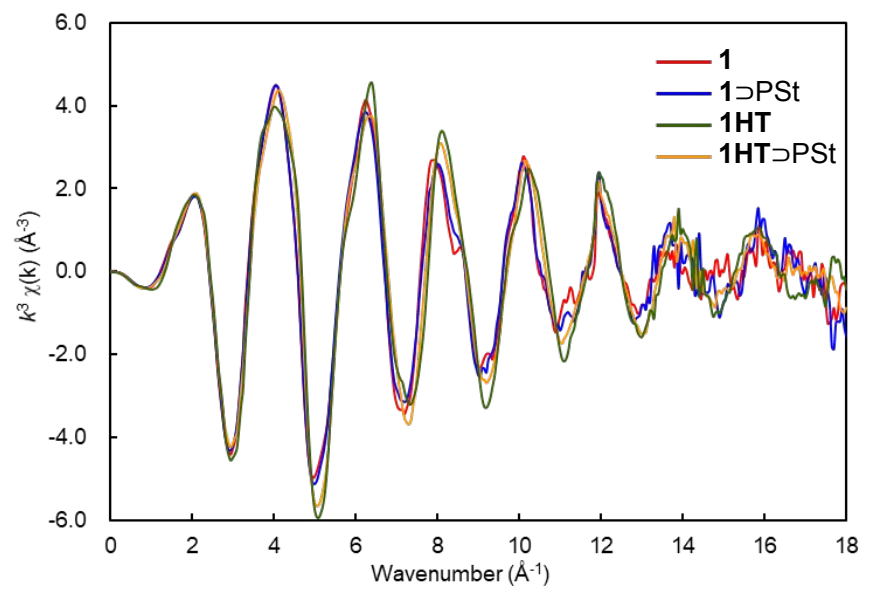

Figure S6. Zn K-edge EXAFS spectra in $k$-space of 1, 1つPSt, 1HT, and 1HTつPSt.

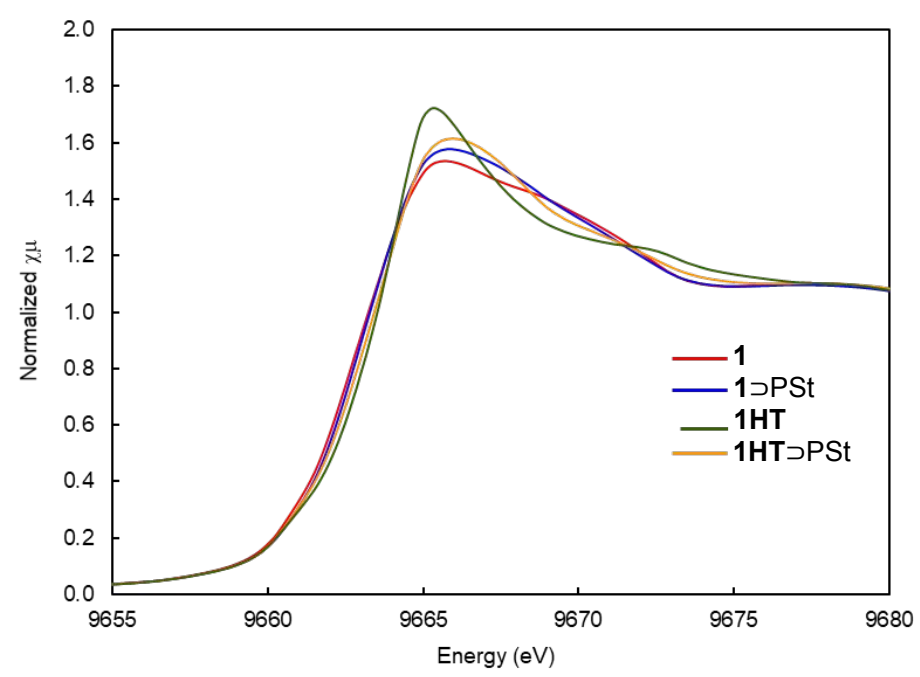

Figure S7. Zn K-edge XANES spectra of 1, 1دPSt, 1HT, and 1HTつPSt. 


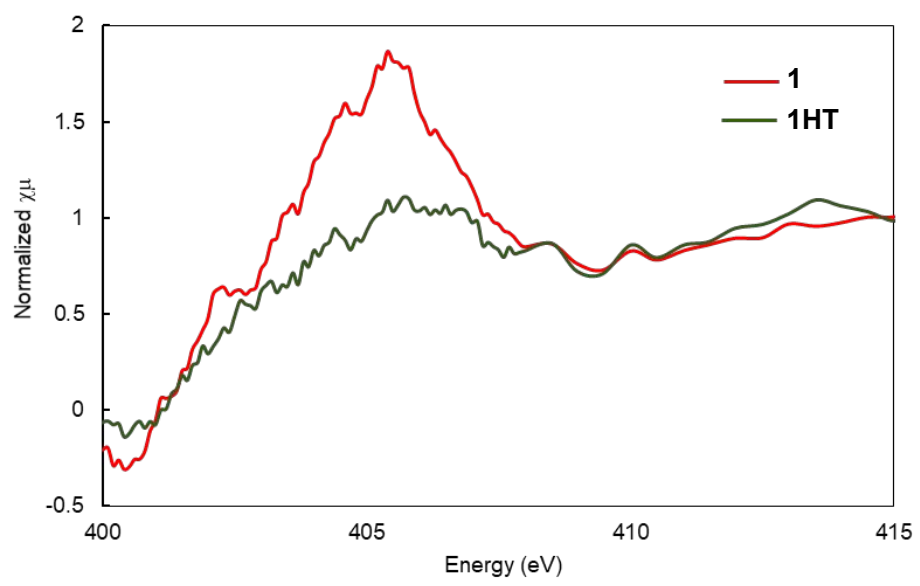

Figure S8. N K-edge NEXAFS spectra of $\mathbf{1}$ and $\mathbf{1 H T}$.

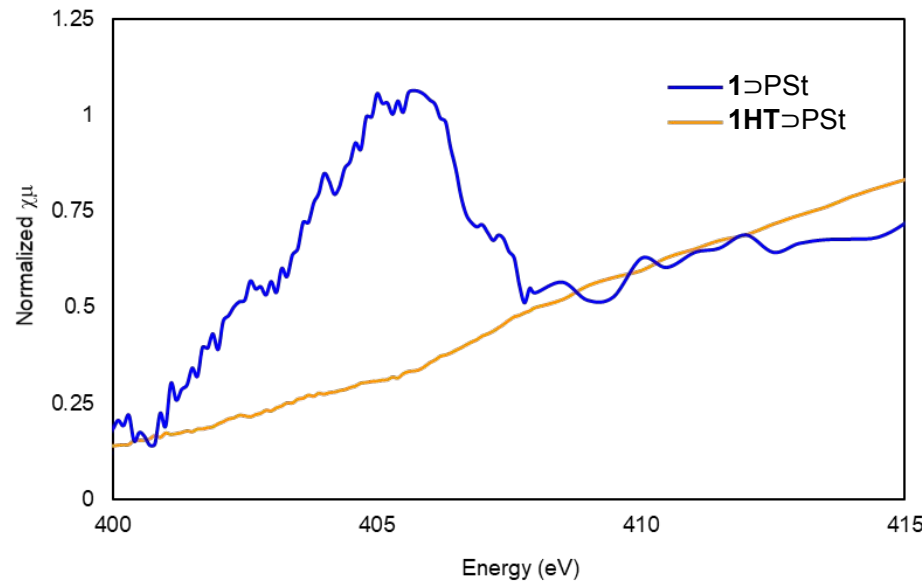

Figure S9. N K-edge XANES spectra of $1 \supset$ PSt and 1HTつPSt. 


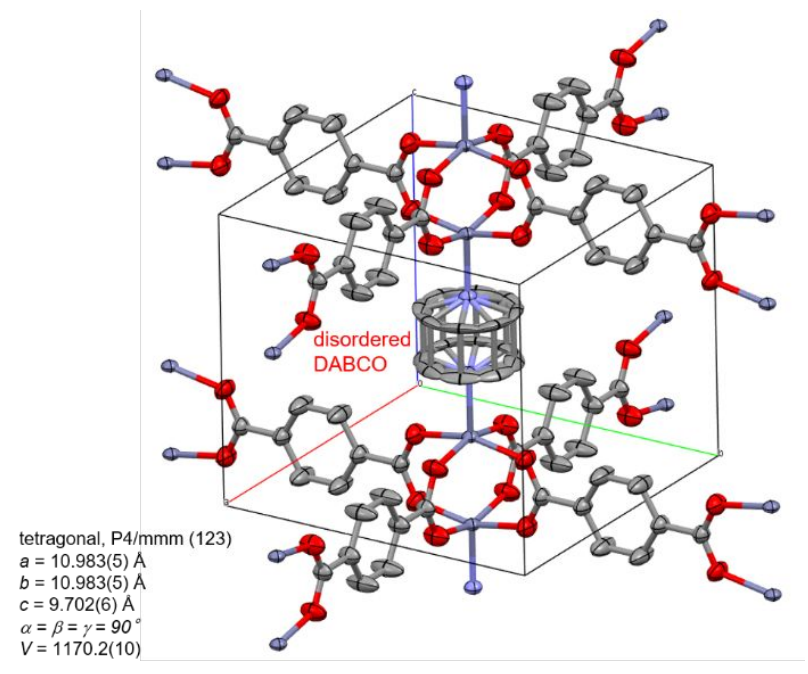

Figure S10. The single crystal X-ray structure of 1 (ORTEP drawing, hydrogen atoms are not shown $)$ with disordering axial DABCO ligand $\left(R_{\text {all }}=0.0672, w R_{\text {all }}^{2}=0.2306, \mathrm{GoF}=0.957\right)$.

Table S1. Selected atomic distances and bond angles in the molecular structures in single crystal X-ray 1 (Figure S10), DFT-optimized 1 (Figure S11), and non-DABCO-pillared 1HT (Figure S12).

\begin{tabular}{|c|c|c|c|c|c|c|c|c|c|c|c|}
\hline & \multicolumn{6}{|c|}{ Atomic distance $(\AA)$} & \multicolumn{5}{|c|}{ Bond angle $\left({ }^{\circ}\right)$} \\
\hline & $\mathrm{Zn} 1-\mathrm{O} 1$ & $\mathrm{Zn} 1-\mathrm{N} 1$ & $Z n 1-Z n 2$ & O1-C1 & $\mathrm{C} 1-\mathrm{C} 2 \mathrm{Ar}$ & $\begin{array}{c}\text { Zn1-plane } \\
\text { (01O2O3O4) }\end{array}$ & Zn1-O1-C1 & O1-C1-O5 & O1-Zn1-N1 & O1-Zn1-O2 & O1-Zn1-O4 \\
\hline X-ray CIF 1 & 2.040 & 2.099 & 2.936 & 1.251 & 1.509 & 0.351 & 126.69 & 126.45 & 99.92 & 88.30 & 88.30 \\
\hline DFT optimized $\mathbf{1}^{\text {a }}$ & $\begin{array}{l}2.050^{\mathrm{b}} \\
2.055^{\mathrm{b}} \\
2.059^{\mathrm{b}}\end{array}$ & 2.125 & 2.870 & 1.273 & $\begin{array}{l}1.495^{\mathrm{c}} \\
1.496^{\mathrm{c}}\end{array}$ & 0.297 & $\begin{array}{l}124.94^{\mathrm{d}} \\
125.00^{\mathrm{d}}\end{array}$ & $\begin{array}{l}126.67^{\mathrm{e}} \\
126.72^{\mathrm{e}}\end{array}$ & $\begin{array}{l}99.13^{9} \\
99.88^{9}\end{array}$ & 88.33 & 87.98 \\
\hline non-DABCO-pillared 1 & 2.059 & - & 2.567 & 1.282 & 1.511 & 0.145 & 121.35 & 125.4 & - & 89.71 & 89.71 \\
\hline
\end{tabular}

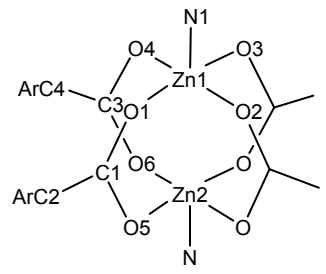

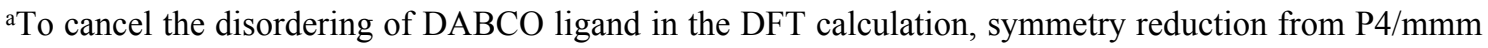
(\#123) of the original crystal system to Pm (\#6) was performed. ${ }^{b} \mathrm{Zn} 1-\mathrm{O} 1, \mathrm{Zn1-O} / \mathrm{Zn1-O} 4, \mathrm{Zn1}-\mathrm{O} 3 .{ }^{\mathrm{c}} \mathrm{C} 1-$

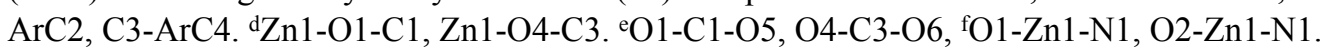




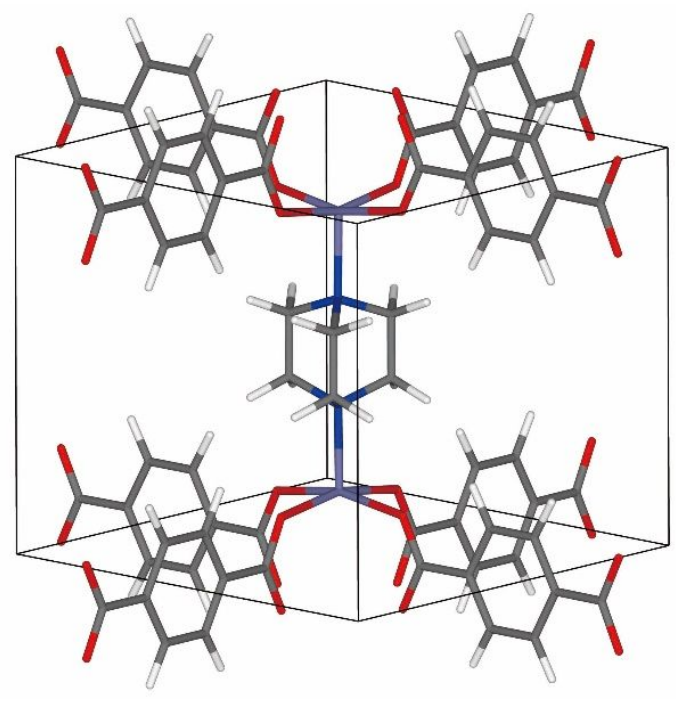

Figure S11. DFT-optimized molecular geometry of $\mathbf{1}$ using the single crystal X-ray structure as the initial geometry. The molecular geometry optimization was carried out by CASTEP code with maintaining the original lattice parameter. 
Table S2. Atomic coordinate of the molecular geometry of $\mathbf{1}$ in Figure S11.

\begin{tabular}{|c|c|c|c|c|c|c|c|c|c|c|c|}
\hline & \multicolumn{3}{|c|}{ Atomic Coordinate } & & \multicolumn{3}{|c|}{ Atomic Coordinate } & & \multicolumn{3}{|c|}{ Atomic Coordinate } \\
\hline Symbol & $\mathrm{x}$ & (2) & $z$ & Symbol & $\mathrm{x}$ & 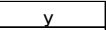 & $z$ & Symbol & $x$ & 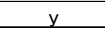 & $z$ \\
\hline $\mathrm{Zn}$ & 5.566074 & 1.435217 & 5.533016 & $\mathrm{C}$ & 1.461947 & 9.702000 & 5.508524 & $\mathrm{H}$ & 5.480187 & 2.152292 & -1.216368 \\
\hline $\mathrm{Zn}$ & 5.566074 & 8.266783 & 5.533016 & $\mathrm{C}$ & 8.165860 & 0.000000 & 5.546305 & $\mathrm{C}$ & 5.485679 & 0.000000 & -1.351568 \\
\hline 0 & 3.528289 & 1.137948 & 5.525986 & $\mathrm{C}$ & 8.165860 & 9.702000 & 5.546305 & $\mathrm{H}$ & 5.480187 & 7.549708 & -1.216368 \\
\hline 0 & 3.528289 & 8.564052 & 5.525986 & $\mathrm{C}$ & 9.659109 & 0.000000 & 5.523241 & $\mathrm{C}$ & 5.485679 & 9.702000 & $\begin{array}{l}-1.351568 \\
\end{array}$ \\
\hline $\mathrm{N}$ & 5.624943 & 3.559567 & 5.504680 & $\mathrm{C}$ & 9.659109 & 9.702000 & 5.523241 & $\mathrm{C}$ & -0.626580 & -1.219444 & 5.513686 \\
\hline $\mathrm{N}$ & 5.624943 & 6.142433 & 5.504680 & C & 5.514345 & 0.000000 & 8.136975 & $\mathrm{H}$ & 1.324989 & -2.153068 & 5.501604 \\
\hline $\mathrm{C}$ & 0.763648 & 1.219541 & 5.506107 & $\mathrm{C}$ & 5.514345 & 9.702000 & 8.136975 & $\mathrm{C}$ & -0.626580 & 10.921444 & 5.513686 \\
\hline C & 0.763648 & 8.482458 & 5.506107 & C & 5.485679 & 0.000000 & 9.631432 & $\mathrm{H}$ & 1.324989 & 11.855067 & 5.501604 \\
\hline $\mathrm{H}$ & 1.324989 & 2.153068 & 5.501604 & C & 5.485679 & 9.702000 & 9.631432 & $\mathrm{H}$ & 9.792662 & -2.151807 & 5.515772 \\
\hline $\mathrm{H}$ & 1.324989 & 7.548932 & 5.501604 & $\mathrm{C}$ & 5.536860 & 0.000000 & 2.928617 & $\mathrm{C}$ & 11.746648 & -1.219542 & 5.506107 \\
\hline C & 4.321481 & 4.076101 & 4.975299 & C & 5.536860 & 9.702000 & 2.928617 & $\mathrm{H}$ & 9.792662 & 11.853806 & 5.515772 \\
\hline C & 4.321481 & 5.625898 & 4.975299 & C & 5.496772 & 0.000000 & 1.434600 & $\mathrm{C}$ & 11.746648 & 10.921541 & 5.506107 \\
\hline 0 & 7.594195 & 1.137560 & 5.555421 & $\mathrm{C}$ & 5.496772 & 9.702000 & 1.434600 & $\mathrm{H}$ & 5.480187 & -2.152292 & 9.766632 \\
\hline 0 & 7.594195 & 8.564440 & 5.555421 & $\mathrm{C}$ & -0.626580 & 1.219444 & 5.513686 & $\mathrm{C}$ & 5.485569 & -1.219736 & 11.719740 \\
\hline C & 10.356420 & 1.219444 & 5.513686 & C & -0.626580 & 8.482555 & 5.513686 & $\mathrm{H}$ & 5.480187 & 11.854291 & 9.766632 \\
\hline C & 10.356420 & 8.482555 & 5.513686 & C & 11.746648 & 1.219541 & 5.506107 & $\mathrm{C}$ & 5.485569 & 10.921735 & 11.719740 \\
\hline $\mathrm{H}$ & 9.792662 & 2.151807 & 5.515772 & $\mathrm{C}$ & 11.746648 & 8.482458 & 5.506107 & $\mathrm{C}$ & 5.480407 & -1.219542 & -0.653708 \\
\hline $\mathrm{H}$ & 9.792662 & 7.550193 & 5.515772 & C & 5.485569 & 1.219735 & 11.719740 & $\mathrm{H}$ & 5.487656 & -2.152874 & $\begin{array}{l}1.299618 \\
\end{array}$ \\
\hline C & 6.735544 & 4.073967 & 4.637791 & C & 5.485569 & 8.482264 & 11.719740 & $\mathrm{C}$ & 5.480407 & 10.921541 & -0.653708 \\
\hline$C$ & 6.735544 & 5.628033 & 4.637791 & $\mathrm{C}$ & 5.480407 & 1.219541 & -0.653708 & $\mathrm{H}$ & 5.487656 & 11.854874 & $\begin{array}{l}1.299618 \\
\end{array}$ \\
\hline C & 5.821869 & 4.076392 & 6.897654 & C & 5.480407 & 8.482458 & -0.653708 & $\mathrm{C}$ & -2.817140 & 0.000000 & 5.546305 \\
\hline $\mathrm{C}$ & 5.821869 & 5.625607 & 6.897654 & 0 & 3.528289 & \begin{tabular}{|l|}
-1.137948 \\
\end{tabular} & 5.525986 & $C$ & $\begin{array}{l}-2.817140 \\
\end{array}$ & 9.702000 & 5.546305 \\
\hline 0 & 5.529282 & 1.137268 & 7.566079 & 0 & 3.528289 & 10.839947 & 5.525986 & C & 13.940502 & 0.000000 & 5.520495 \\
\hline 0 & 5.529282 & 8.564732 & $\begin{array}{l}.566079 \\
\end{array}$ & C & 0.763648 & -1.219542 & 5.506107 & $\mathrm{C}$ & 13.940502 & 9.702000 & 5.520495 \\
\hline C & 5.480407 & 1.219541 & 10.329291 & C & 0.763648 & 10.921541 & 5.506107 & C & 5.536860 & 0.000000 & 13.911616 \\
\hline $\mathrm{C}$ & 5.480407 & 8.482458 & 10.329291 & 0 & 7.594195 & -1.137560 & 5.555421 & $\mathrm{C}$ & 5.536860 & 9.702000 & 13.911616 \\
\hline $\mathrm{H}$ & 5.480187 & 2.152292 & 9.766632 & 0 & 7.594195 & 10.839560 & 5.555421 & $\mathrm{C}$ & 5.514345 & 0.000000 & -2.846025 \\
\hline $\mathrm{H}$ & 5.480187 & 7.549708 & 9.766632 & C & 10.356420 & -1.219444 & 5.513686 & $C$ & 5.514345 & 9.702000 & -2.846025 \\
\hline 0 & 5.555751 & 1.138530 & 3.499843 & $\mathrm{C}$ & 10.356420 & 10.921444 & 5.513686 & $\mathrm{H}$ & $\begin{array}{l}-1.190338 \\
\end{array}$ & -2.151807 & 5.515772 \\
\hline 0 & 5.555751 & 8.563470 & 3.499843 & 0 & 5.529282 & -1.137268 & 7.566079 & $\mathrm{H}$ & -1.190338 & 11.853806 & 5.515772 \\
\hline C & 5.485569 & 1.219735 & 0.736740 & 0 & 5.529282 & 10.839268 & $\begin{array}{l}7.566079 \\
\end{array}$ & $\mathrm{H}$ & 12.307989 & -2.153068 & 5.501604 \\
\hline C & 5.485569 & 8.482264 & 0.736740 & C & 5.480407 & -1.219542 & 10.329291 & $\mathrm{H}$ & 12.307989 & 11.855067 & 5.501604 \\
\hline $\mathrm{H}$ & 5.487656 & 2.152874 & 1.299618 & C & 5.480407 & 10.921541 & 10.329291 & $\mathrm{H}$ & 5.487656 & -2.152874 & 12.282619 \\
\hline $\mathrm{H}$ & 5.487656 & 7.549126 & 1.299618 & 0 & 5.555751 & -1.138530 & 3.499843 & $\mathrm{H}$ & 5.487656 & 11.854874 & 12.282619 \\
\hline $\mathrm{H}$ & 3.524115 & 3.672304 & 5.609677 & 0 & 5.555751 & 10.840529 & 3.499843 & $\mathrm{H}$ & 5.480187 & -2.152292 & -1.216368 \\
\hline $\mathrm{H}$ & 3.524115 & 6.029696 & 5.609677 & C & 5.485569 & -1.219736 & 0.736740 & $\mathrm{H}$ & 5.480187 & 11.854291 & -1.216368 \\
\hline $\mathrm{H}$ & 4.182546 & 3.660468 & 3.969037 & $\mathrm{C}$ & 5.485569 & 10.921735 & 0.736740 & 0 & -3.388804 & -1.137560 & 5.555421 \\
\hline $\mathrm{H}$ & 4.182546 & 6.041532 & 3.969037 & $\mathrm{H}$ & $\begin{array}{l}-1.190338 \\
\end{array}$ & 2.151807 & 5.515772 & 0 & -3.388804 & $\begin{array}{l}1.137560 \\
\end{array}$ & 5.555421 \\
\hline $\mathrm{H}$ & 6.590129 & 3.660758 & 3.631199 & $\mathrm{C}$ & -1.323891 & 0.000000 & 5.523241 & 0 & -3.388804 & 8.564440 & 5.555421 \\
\hline $\mathrm{H}$ & 6.590129 & 6.041241 & 3.631199 & $\mathrm{H}$ & -1.190338 & 7.550193 & 5.515772 & 0 & -3.388804 & 10.839560 & 5.555421 \\
\hline $\mathrm{H}$ & 7.676458 & 3.664542 & 5.025821 & C & -1.323891 & 9.702000 & 5.523241 & 0 & 14.511289 & -1.137948 & 5.525986 \\
\hline $\mathrm{H}$ & 7.676458 & 6.037457 & 5.025821 & $\mathrm{H}$ & 12.307989 & 2.153068 & 5.501604 & 0 & 14.511289 & 1.137948 & 5.525986 \\
\hline $\mathrm{H}$ & 6.765418 & 3.663475 & 7.277884 & C & 12.444947 & 0.000000 & 5.508524 & 0 & 14.511289 & 8.564052 & 5.525986 \\
\hline $\mathrm{H}$ & 6.765418 & 6.038525 & 7.277884 & $\mathrm{H}$ & 12.307989 & 7.548932 & 5.501604 & 0 & 14.511289 & 10.839947 & 5.525986 \\
\hline $\mathrm{H}$ & 5.014838 & 3.670655 & 7.519840 & C & 12.444947 & 9.702000 & 5.508524 & 0 & 5.555751 & -1.138530 & 14.482842 \\
\hline $\mathrm{H}$ & 5.014838 & 6.031345 & 7.519840 & $\mathrm{H}$ & 5.487656 & 2.152874 & 12.282619 & 0 & 5.555751 & 1.138530 & 14.482842 \\
\hline C & 2.957502 & 0.000000 & 5.520495 & C & 5.496772 & 0.000000 & 12.417600 & 0 & 5.555751 & 8.563470 & 14.482842 \\
\hline C & 2.957502 & 9.702000 & 5.520495 & $\mathrm{H}$ & 5.487656 & 7.549126 & 12.282619 & 0 & 5.555751 & 10.840529 & 14.482842 \\
\hline $\mathrm{C}$ & 1.461947 & 0.000000 & 5.508524 & $\mathrm{C}$ & 5.496772 & 9.702000 & 12.417600 & 0 & 5.529282 & -1.137268 & -3.416921 \\
\hline & & & & & & & & 0 & 5.529282 & 1.137268 & -3.416921 \\
\hline & & & & & & & & 0 & 5.529282 & $\begin{array}{l}8.564732 \\
\end{array}$ & -3.416921 \\
\hline & & & & & & & & 0 & 5.529282 & 10.839268 & -3.416921 \\
\hline
\end{tabular}




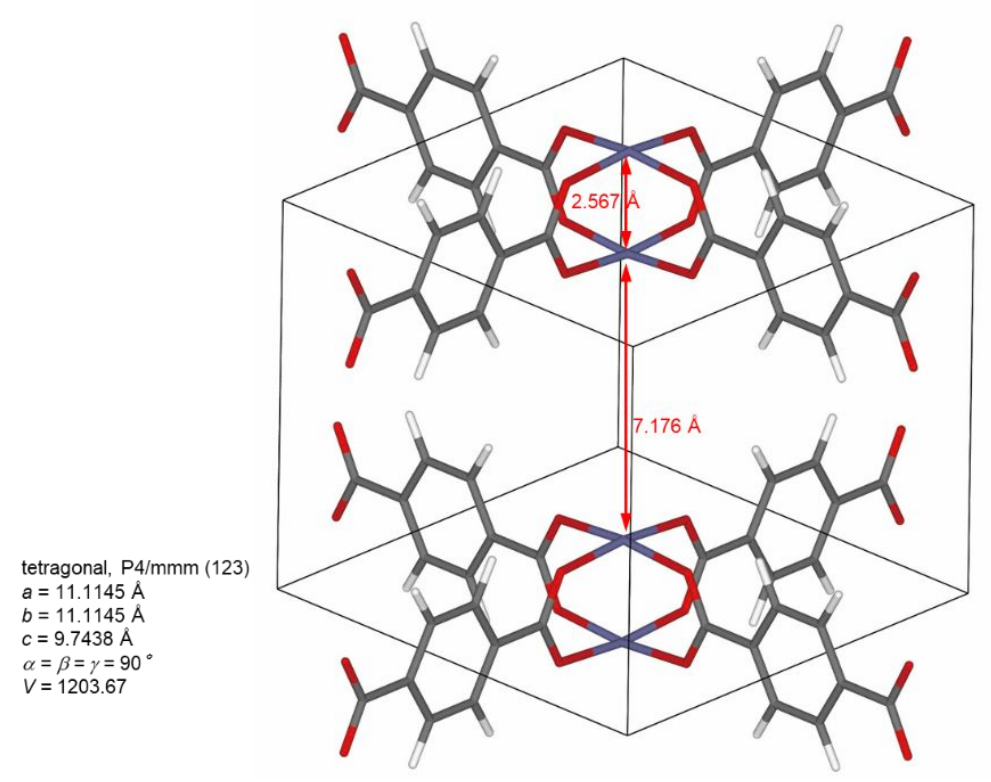

Figure S12. DFT-optimized molecular geometry of non-DABCO-pillared 1HT. The optimization of cell parameter was simultaneously performed with the molecular geometry optimization. 
Table S3. Atomic coordinate of the molecular geometry of $\mathbf{1 H T}$ in Figure S12.

\begin{tabular}{|c|r|r|r|}
\hline & \multicolumn{3}{|c|}{ Atomic Coordinate } \\
\hline Symbol & x & $y$ & $z$ \\
\hline $\mathrm{Zn}$ & 5.557250 & 5.557250 & 1.283746 \\
\hline $\mathrm{Zn}$ & 5.557250 & 5.557250 & 8.460054 \\
\hline $\mathrm{O}$ & 5.557250 & 3.511960 & 1.138953 \\
\hline $\mathrm{O}$ & 5.557250 & 7.602541 & 8.604847 \\
\hline $\mathrm{O}$ & 5.557250 & 7.602541 & 1.138953 \\
\hline $\mathrm{O}$ & 5.557250 & 3.511960 & 8.604847 \\
\hline $\mathrm{O}$ & 7.602541 & 5.557250 & 1.138953 \\
\hline $\mathrm{O}$ & 3.511960 & 5.557250 & 8.604847 \\
\hline $\mathrm{O}$ & 3.511960 & 5.557250 & 1.138953 \\
\hline $\mathrm{O}$ & 7.602541 & 5.557250 & 8.604847 \\
\hline $\mathrm{C}$ & 5.557250 & 0.698991 & 1.216319 \\
\hline $\mathrm{C}$ & 5.557250 & 10.415509 & 8.527481 \\
\hline $\mathrm{C}$ & 5.557250 & 10.415509 & 1.216319 \\
\hline $\mathrm{C}$ & 5.557250 & 0.698991 & 8.527481 \\
\hline $\mathrm{C}$ & 10.415509 & 5.557250 & 1.216319 \\
\hline $\mathrm{C}$ & 0.698991 & 5.557250 & 8.527481 \\
\hline $\mathrm{C}$ & 0.698991 & 5.557250 & 1.216319 \\
\hline $\mathrm{C}$ & 10.415509 & 5.557250 & 8.527481 \\
\hline $\mathrm{H}$ & 5.557250 & 1.248714 & 2.157959 \\
\hline $\mathrm{H}$ & 5.557250 & 9.865787 & 7.585841 \\
\hline $\mathrm{H}$ & 5.557250 & 9.865787 & 2.157959 \\
\hline $\mathrm{H}$ & 5.557250 & 1.248714 & 7.585841 \\
\hline $\mathrm{H}$ & 9.865787 & 5.557250 & 2.157959 \\
\hline $\mathrm{H}$ & 1.248714 & 5.557250 & 7.585841 \\
\hline $\mathrm{H}$ & 1.248714 & 5.557250 & 2.157959 \\
\hline $\mathrm{H}$ & 9.865787 & 5.557250 & 7.585841 \\
\hline $\mathrm{C}$ & 5.557250 & 2.924114 & 0.000000 \\
\hline $\mathrm{C}$ & 5.557250 & 2.924114 & 9.743800 \\
\hline $\mathrm{C}$ & 5.557250 & 8.190386 & 0.000000 \\
\hline $\mathrm{C}$ & 5.557250 & 8.190386 & 9.743800 \\
\hline $\mathrm{C}$ & 8.190386 & 5.557250 & 0.000000 \\
\hline $\mathrm{C}$ & 8.190386 & 5.557250 & 9.743800 \\
\hline $\mathrm{C}$ & 2.924114 & 5.557250 & 0.000000 \\
\hline $\mathrm{C}$ & 2.924114 & 5.557250 & 9.743800 \\
\hline $\mathrm{C}$ & 5.557250 & 1.412653 & 0.000000 \\
\hline $\mathrm{C}$ & 5.557250 & 1.412653 & 9.743800 \\
\hline $\mathrm{C}$ & 5.557250 & 9.701847 & 0.000000 \\
\hline $\mathrm{C}$ & 5.557250 & 9.701847 & 9.743800 \\
\hline $\mathrm{C}$ & 9.701847 & 5.557250 & 0.000000 \\
\hline $\mathrm{C}$ & 9.701847 & 5.557250 & 9.743800 \\
\hline $\mathrm{C}$ & 1.412653 & 5.557250 & 0.000000 \\
\hline $\mathrm{C}$ & 1.412653 & 5.557250 & 9.743800 \\
\hline $\mathrm{C}$ & 5.557250 & -0.698991 & 1.216319 \\
\hline & & & \\
\hline
\end{tabular}

\begin{tabular}{|c|c|c|c|}
\hline & \multicolumn{3}{|c|}{ Atomic Coordinate } \\
\hline Symbol & $\mathrm{x}$ & $\mathrm{y}$ & $z$ \\
\hline $\mathrm{C}$ & 5.557250 & 11.813491 & 1.216319 \\
\hline $\mathrm{C}$ & 5.557250 & -0.698991 & 8.527481 \\
\hline C & 11.813491 & 5.557250 & 1.216319 \\
\hline C & -0.698991 & 5.557250 & 8.527481 \\
\hline C & -0.698991 & 5.557250 & 1.216319 \\
\hline $\mathrm{C}$ & 11.813491 & 5.557250 & 8.527481 \\
\hline 0 & 5.557250 & 3.511960 & -1.138953 \\
\hline 0 & 5.557250 & 3.511960 & 10.882753 \\
\hline 0 & 5.557250 & 7.602541 & -1.138953 \\
\hline 0 & 5.557250 & 7.602541 & 10.882753 \\
\hline 0 & 7.602541 & 5.557250 & -1.138953 \\
\hline 0 & 7.602541 & 5.557250 & 10.882753 \\
\hline 0 & 3.511960 & 5.557250 & -1.138953 \\
\hline 0 & 3.511960 & 5.557250 & 10.882753 \\
\hline $\mathrm{C}$ & 5.557250 & 0.698991 & -1.216319 \\
\hline $\mathrm{C}$ & 5.557250 & 0.698991 & 10.960119 \\
\hline $\mathrm{C}$ & 5.557250 & 10.415509 & -1.216319 \\
\hline $\mathrm{C}$ & 5.557250 & 10.415509 & 10.960119 \\
\hline $\mathrm{C}$ & 10.415509 & 5.557250 & -1.216319 \\
\hline C & 10.415509 & 5.557250 & 10.960119 \\
\hline$C$ & 0.698991 & 5.557250 & -1.216319 \\
\hline $\mathrm{C}$ & 0.698991 & 5.557250 & 10.960119 \\
\hline $\mathrm{H}$ & 5.557250 & -1.248713 & 2.157959 \\
\hline C & 5.557250 & -1.412653 & 0.000000 \\
\hline $\mathrm{H}$ & 5.557250 & 12.363214 & 7.585841 \\
\hline $\mathrm{C}$ & 5.557250 & 12.527153 & 9.743800 \\
\hline $\mathrm{H}$ & 5.557250 & 12.363214 & 2.157959 \\
\hline C & 5.557250 & 12.527153 & 0.000000 \\
\hline $\mathrm{H}$ & 5.557250 & -1.248713 & 7.585841 \\
\hline C & 5.557250 & -1.412653 & 9.743800 \\
\hline $\mathrm{H}$ & 12.363214 & 5.557250 & 2.157959 \\
\hline $\mathrm{C}$ & 12.527153 & 5.557250 & 0.000000 \\
\hline $\mathrm{H}$ & -1.248713 & 5.557250 & 7.585841 \\
\hline $\mathrm{C}$ & -1.412653 & 5.557250 & 9.743800 \\
\hline $\mathrm{H}$ & -1.248713 & 5.557250 & 2.157959 \\
\hline $\mathrm{C}$ & -1.412653 & 5.557250 & 0.000000 \\
\hline $\mathrm{H}$ & 12.363214 & 5.557250 & 7.585841 \\
\hline C & 12.527153 & 5.557250 & 9.743800 \\
\hline C & 5.557250 & -0.698991 & -1.216319 \\
\hline $\mathrm{H}$ & 5.557250 & 1.248714 & -2.157959 \\
\hline $\mathrm{C}$ & 5.557250 & -0.698991 & 10.960119 \\
\hline $\mathrm{H}$ & 5.557250 & 1.248714 & 11.901760 \\
\hline $\mathrm{H}$ & 5.557250 & 9.865787 & -2.157959 \\
\hline $\mathrm{C}$ & 5.557250 & 11.813491 & -1.216319 \\
\hline
\end{tabular}

\begin{tabular}{|r|r|r|r|}
\hline & \multicolumn{3}{|c|}{ Atomic Coordinate } \\
\hline Symbol & \multicolumn{1}{|c|}{$\mathrm{x}$} & $\mathrm{y}$ & $\mathrm{z}$ \\
\hline $\mathrm{H}$ & 5.557250 & 9.865787 & 11.901760 \\
\hline $\mathrm{C}$ & 5.557250 & 11.813491 & 10.960119 \\
\hline $\mathrm{H}$ & 9.865787 & 5.557250 & -2.157959 \\
\hline $\mathrm{C}$ & 11.813491 & 5.557250 & -1.216319 \\
\hline $\mathrm{H}$ & 9.865787 & 5.557250 & 11.901760 \\
\hline $\mathrm{C}$ & 11.813491 & 5.557250 & 10.960119 \\
\hline $\mathrm{C}$ & -0.698991 & 5.557250 & -1.216319 \\
\hline $\mathrm{H}$ & 1.248714 & 5.557250 & -2.157959 \\
\hline $\mathrm{C}$ & -0.698991 & 5.557250 & 10.960119 \\
\hline $\mathrm{H}$ & 1.248714 & 5.557250 & 11.901760 \\
\hline $\mathrm{C}$ & 5.557250 & -2.924114 & 0.000000 \\
\hline $\mathrm{C}$ & 5.557250 & 14.038614 & 9.743800 \\
\hline $\mathrm{C}$ & 5.557250 & 14.038614 & 0.000000 \\
\hline $\mathrm{C}$ & 5.557250 & -2.924114 & 9.743800 \\
\hline $\mathrm{C}$ & 14.038614 & 5.557250 & 0.000000 \\
\hline $\mathrm{C}$ & -2.924114 & 5.557250 & 9.743800 \\
\hline $\mathrm{C}$ & -2.924114 & 5.557250 & 0.000000 \\
\hline $\mathrm{C}$ & 14.038614 & 5.557250 & 9.743800 \\
\hline $\mathrm{H}$ & 5.557250 & -1.248713 & -2.157959 \\
\hline $\mathrm{H}$ & 5.557250 & -1.248713 & 11.901760 \\
\hline $\mathrm{H}$ & 5.557250 & 12.363214 & -2.157959 \\
\hline $\mathrm{H}$ & 5.557250 & 12.363214 & 11.901760 \\
\hline $\mathrm{H}$ & 12.363214 & 5.557250 & -2.157959 \\
\hline $\mathrm{H}$ & 12.363214 & 5.557250 & 11.901760 \\
\hline $\mathrm{H}$ & -1.248713 & 5.557250 & -2.157959 \\
\hline $\mathrm{H}$ & -1.248713 & 5.557250 & 11.901760 \\
\hline $\mathrm{O}$ & 5.557250 & -3.511959 & -1.138953 \\
\hline $\mathrm{O}$ & 5.557250 & -3.511959 & 1.138953 \\
\hline $\mathrm{O}$ & 5.557250 & 14.626459 & 8.604847 \\
\hline $\mathrm{O}$ & 5.557250 & 14.626459 & 10.882753 \\
\hline $\mathrm{O}$ & 5.557250 & 14.626459 & -1.138953 \\
\hline $\mathrm{O}$ & 5.557250 & 14.626459 & 1.138953 \\
\hline $\mathrm{O}$ & 5.557250 & -3.511959 & 8.604847 \\
\hline $\mathrm{O}$ & 5.557250 & -3.511959 & 10.882753 \\
\hline $\mathrm{O}$ & 14.626459 & 5.557250 & -1.138953 \\
\hline $\mathrm{O}$ & 14.626459 & 5.557250 & 1.138953 \\
\hline $\mathrm{O}$ & -3.511959 & 5.557250 & 8.604847 \\
\hline $\mathrm{O}$ & -3.511959 & 5.557250 & 10.882753 \\
\hline $\mathrm{O}$ & -3.511959 & 5.557250 & -1.138953 \\
\hline $\mathrm{O}$ & -3.511959 & 5.557250 & 1.138953 \\
\hline $\mathrm{O}$ & 14.626459 & 5.557250 & 8.604847 \\
\hline & 5.557250 & 5.557250 & 11.027546 \\
\hline $\mathrm{H}$ & 5.557250 & 5.557250 & -1.283746 \\
\hline
\end{tabular}


The corresponding XAS data were processed using Athena by extracting the EXAFS oscillations $\chi(k)$ as a function of photoelectron wave number $k$. Fourier transformation of the $k^{3}$-weighted $\chi$ from $k$ space to $r$ space was carried out to obtain the radial distribution function. The EXAFS fitting calculation was performed by FEFF $6^{2}$ program embedded with Artemis, where the theoretical scattering paths were generated from crystallographic coordinate based DFT-optimized DABCO-pillared X-ray structure of 1 (Figure S11, Table S2), and DFT-calculated non-DABCO-pillared structure of 1HT. The parameters for FEFF fitting analysis are as follows: Ab-Sc: the X-ray absorbing atom and the scattering atom; $C N$ : coordination number; $D W$ : Debye-Waller factor $\left(\AA^{2}\right) ; \Delta E$ : energy shift $(\mathrm{eV}) ; R$ : atomic distance $(\AA)$. The parameter for the many-body effect of $S_{0}{ }^{2}$ is fixed to 1.0 for all fitting calculations.

For fitting calculation of 1 based on the $X$-ray structure, six independent parameters (Zn-O1, Zn-N: $S_{0}^{2}$, e1, r1, o1; Zn-Zn, Zn-C1 $\left(\mathrm{CO}_{2}\right)$, Zn-C2(DABCO), Zn-O1-C1, Zn-O2: $S_{0}{ }^{2}$, e2(=e1), r2, б2) with seven scattering paths (1st shell: Zn-O1, Zn-N, 2nd shell: Zn$\left.\mathrm{Zn}, \mathrm{Zn}-\mathrm{C} 1\left(\mathrm{CO}_{2}\right), \mathrm{Zn}-\mathrm{C} 2(\mathrm{DABCO}), \mathrm{Zn}-\mathrm{O} 1-\mathrm{C} 1, \mathrm{Zn}-\mathrm{N}-\mathrm{C} 2, \mathrm{Zn}-\mathrm{O} 2\right)$ give the fitting results with adequate accuracy as shown in Figures S13 and S14, where the many-body effect $S_{0}{ }^{2}$ parameter was fixed with an appropriate value as $S_{0}{ }^{2}=1.1$.

For fitting calculation of 1 based on the DFT-optimized structure, six independent parameters (Zn-O1, Zn-N: $S_{0}^{2}$, e1, r1, o1; Zn-Zn, Zn-C1( $\left.\mathrm{CO}_{2}\right), \mathrm{Zn}-\mathrm{C} 2(\mathrm{DABCO}), \mathrm{Zn}-\mathrm{O} 1-$ C1, Zn-O2: $S_{0}^{2}$, e2(=e1), r2, б2) with six scattering paths (1st shell: Zn-O1, Zn-N, 2nd shell: $\mathrm{Zn}-\mathrm{Zn}, \mathrm{Zn}-\mathrm{C} 1\left(\mathrm{CO}_{2}\right)$, $\left.\mathrm{Zn}-\mathrm{C} 2(\mathrm{DABCO}), \mathrm{Zn}-\mathrm{O} 1-\mathrm{C} 1, \mathrm{Zn}-\mathrm{O} 2\right)$ give the fitting results with adequate accuracy as shown in Figures S15 and S16, where the many-body effect $S_{0}{ }^{2}$ parameter was fixed with an appropriate value as $S_{0}{ }^{2}=1.1$. The refined parameters were summarized in tables with each fitting result.

For fitting calculation of $1 \supset P S t$ based on the X-ray structure, five independent parameters (Zn-O1, Zn-N: $S_{0}^{2}$, e1, r1, o1; Zn-Zn, Zn-C1( $\left.\mathrm{CO}_{2}\right), \mathrm{Zn}-\mathrm{C} 2(\mathrm{DABCO}), \mathrm{Zn}-\mathrm{O} 1-$ $\left.\mathrm{C} 1, \mathrm{Zn}-\mathrm{O} 2: S_{0}{ }^{2}, \mathrm{e} 2(=\mathrm{e} 1), \mathrm{r} 2, \sigma 2\right)$ with seven scattering paths (1 st shell: Zn-O1, Zn-N, 2nd shell: $\left.\mathrm{Zn}-\mathrm{Zn}, \mathrm{Zn}-\mathrm{C} 1\left(\mathrm{CO}_{2}\right), \mathrm{Zn}-\mathrm{C} 2(\mathrm{DABCO}), \mathrm{Zn}-\mathrm{O} 1-\mathrm{C} 1, \mathrm{Zn}-\mathrm{N}-\mathrm{C} 2, \mathrm{Zn}-\mathrm{O} 2\right)$ give the fitting results with adequate accuracy as shown in Figures $\mathrm{S} 17$ and $\mathrm{S} 18$, where the many-body effect $S_{0}^{2}$ parameter was fixed with an appropriate value as $S_{0}{ }^{2}=1.1$

For fitting calculation of $1 \supset \mathrm{PSt}$ based on the DFT-optimized structure, five independent parameters (Zn-O1, Zn-N: $S_{0}^{2}$, e1, r1, o1; Zn-Zn, Zn-C1( $\left.\mathrm{CO}_{2}\right)$, ZnC2(DABCO), Zn-O1-C1, Zn-O2: $S_{0}{ }^{2}$, e2(=e1), r2, o2) with seven scattering paths (1st shell: Zn-O1, Zn-N, 2nd shell: Zn-Zn, Zn-C1 $\left(\mathrm{CO}_{2}\right)$, Zn-C2(DABCO), Zn-O1-C1, Zn-N$\mathrm{C} 2, \mathrm{Zn}-\mathrm{O} 2)$ give the fitting results with adequate accuracy as shown in Figures S19 and 20, where the many-body effect $S_{0}{ }^{2}$ parameter was fixed with an appropriate value as $S_{0}{ }^{2}$ $=0.9$. 


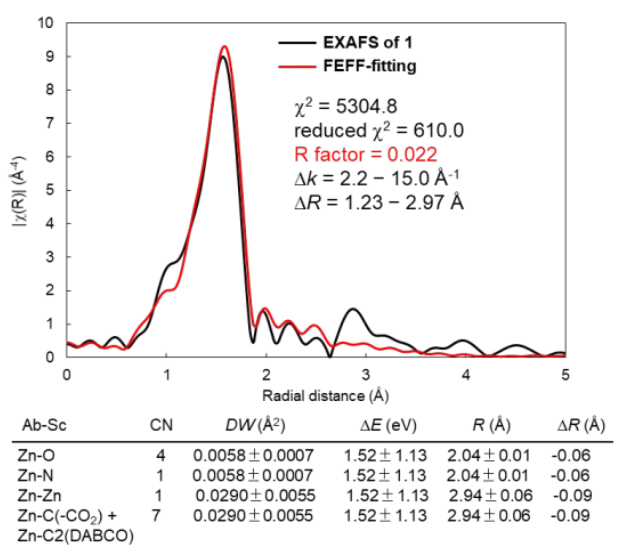

Figure S13. FEFF fitting analysis on the EXAFS spectrum of 1. The FEFF calculation of EXAFS spectrum was carried out using the CIF-based model shown in Figure S10.

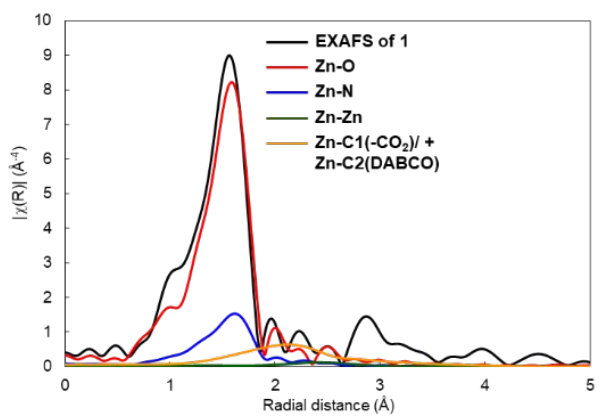

Figure S14. All the scattering paths of the FEFF fitting of Figure S13 on the EXAFS spectrum of 1 . 


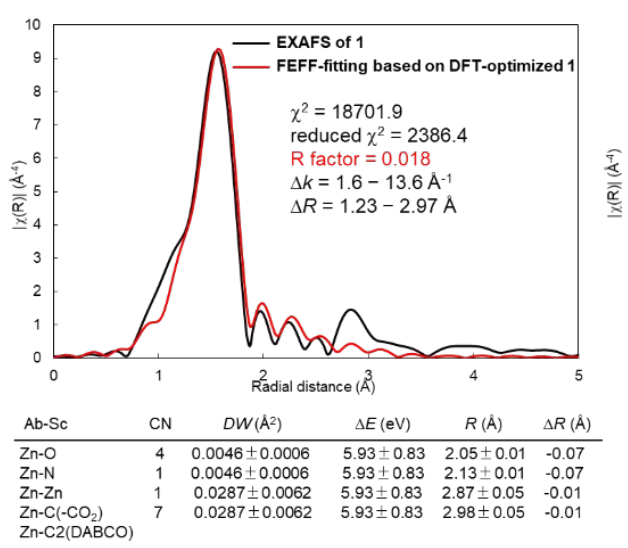

Figure S15. FEFF fitting analysis on the EXAFS spectrum of 1 . The fitting calculation of EXAFS spectrum was carried out using the CIF-based DFT-optimized model shown in Figure S11.

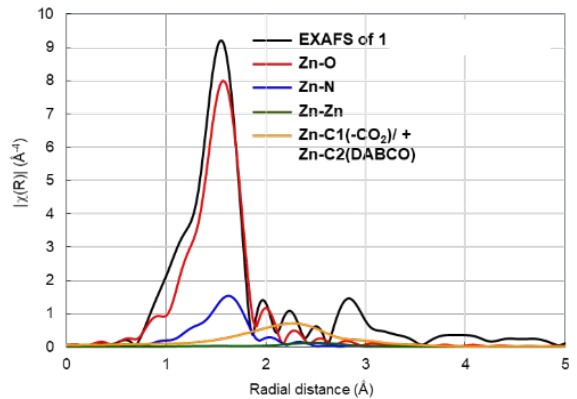

Figure S16. All the scattering paths of the FEFF fitting of Figure S15 on the EXAFS spectrum of 1 . 


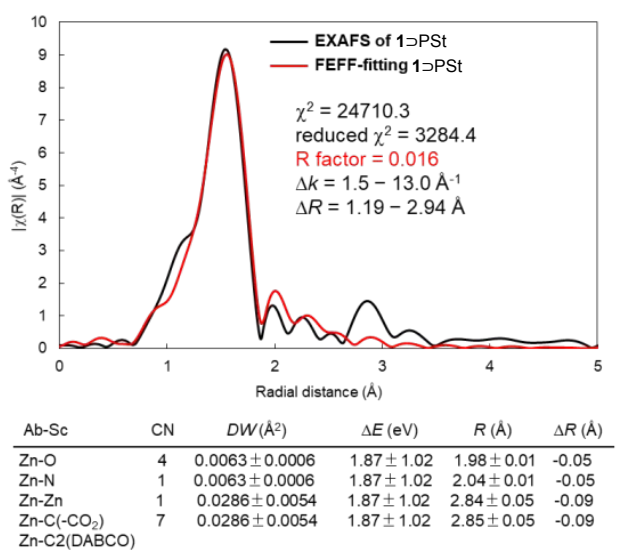

Figure S17. FEFF fitting analysis on the EXAFS spectrum of 1 DPSt. The FEFF calculation of EXAFS spectrum was carried out using the CIF-based model shown in Figure S10.

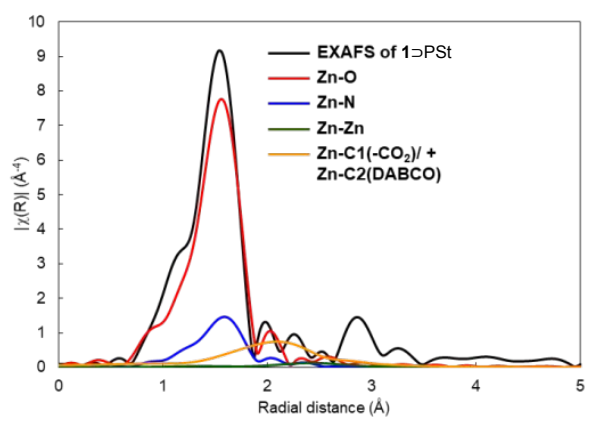

Figure S18. All the scattering paths of the FEFF fitting of Figure S17 on the EXAFS spectrum of $1 \supset$ PSt. 


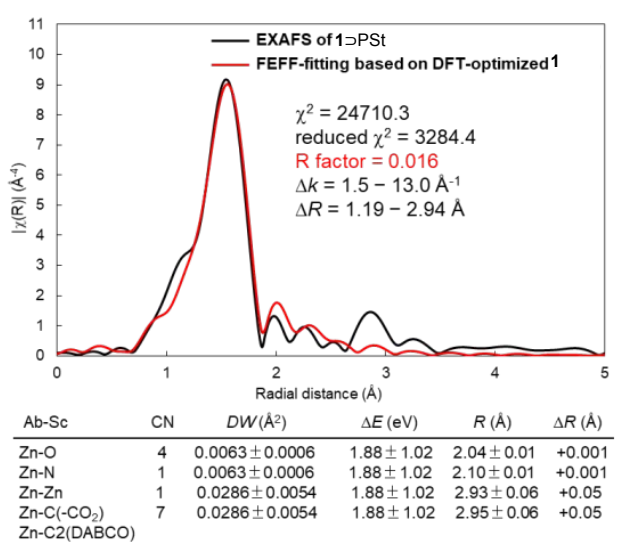

Figure S19. FEFF fitting analysis on the EXAFS spectrum of $1 \supset$ PSt. The fitting calculation of EXAFS spectrum was carried out using the CIF-based DFT-optimized model shown in Figure S11.

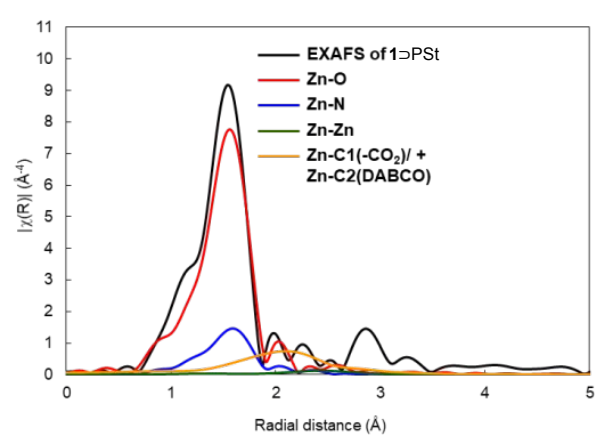

Figure S20. All the scattering paths of the FEFF fitting of Figure S19 on the EXAFS spectrum of $1 \supset$ PSt. 
For fitting calculation of $\mathbf{1 H T}$ (Figure S21 and S22) and 1HTつPSt (Figure S23 and S24), seven independent parameters ( $\mathrm{Zn}-\mathrm{O}: S_{0}^{2}, \mathrm{e} 1, \mathrm{r} 1, \sigma 1 ; \mathrm{Zn}-\mathrm{Zn}, \mathrm{Zn}-\mathrm{C}\left(-\mathrm{CO}_{2}\right): S_{0}^{2}$, e2, r2, $\left.\sigma 2\right)$ with three scattering paths (1st shell: $\mathrm{Zn}-\mathrm{O}, 2 \mathrm{nd}$ shell: $\mathrm{Zn}-\mathrm{Zn}, \mathrm{Zn}-\mathrm{C}\left(-\mathrm{CO}_{2}\right)$ ) give good fitting results as shown in Figures S21-S24, where the many-body effect $S_{0}^{2}$ parameter was fixed with an appropriate value as $S_{0}{ }^{2}=1.0$. The refined parameters were summarized in tables with each fitting result.

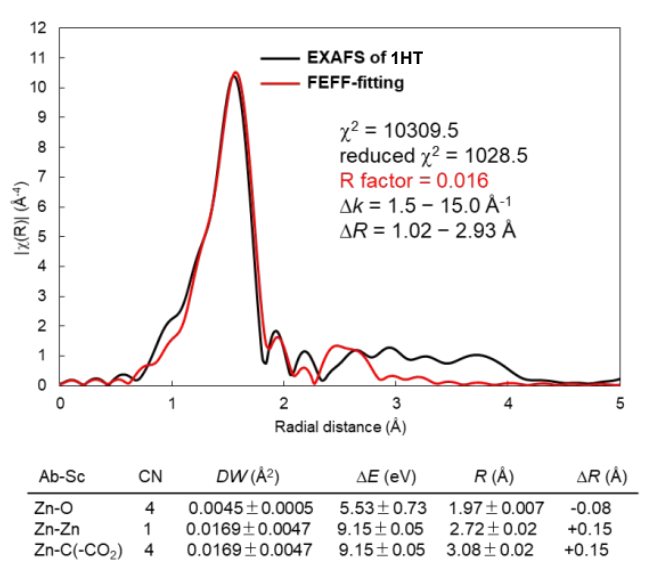

Figure S21. FEFF fitting analysis on the EXAFS spectrum of 1HT. The FEFF calculation of EXAFS spectrum was performed based on the DFT-calculated the non-DABCO-pillared model shown in Figure S12.

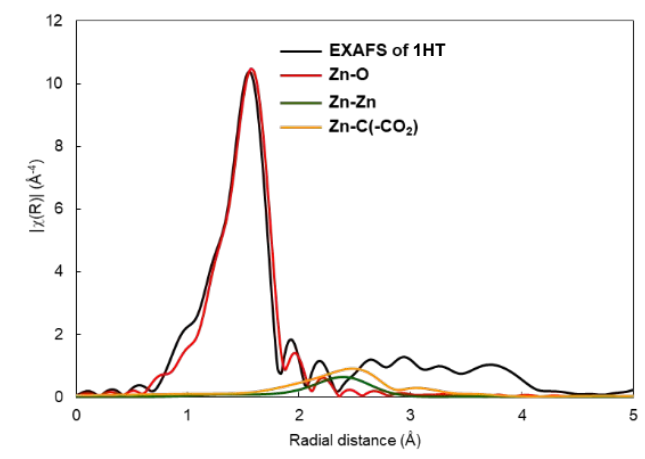

Figure S22. All the scattering paths of the FEFF fitting of Figure S21 on the EXAFS spectrum of $1 \mathrm{HT}$. 


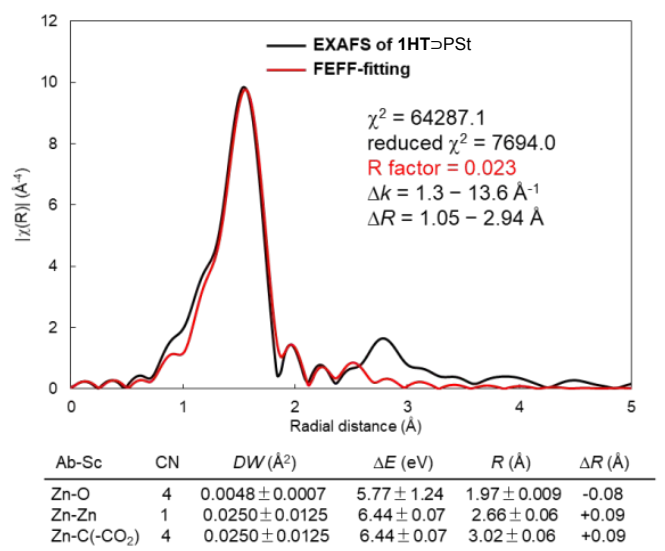

Figure S23. FEFF fitting analysis on the EXAFS spectrum of 1HTつPSt. The FEFF calculation of EXAFS spectrum was performed based on the DFT-calculated the non-DABCO-pillared model shown in Figure S12.

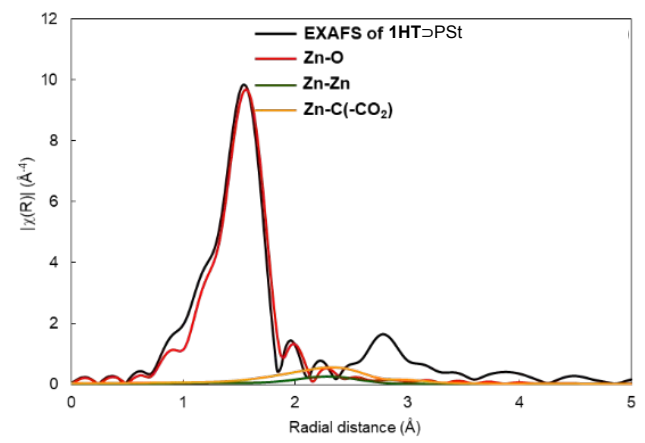

Figure S24. All the scattering paths of the FEFF fitting of Figure S23 on the EXAFS spectrum of 1HT $\supset$ PSt.

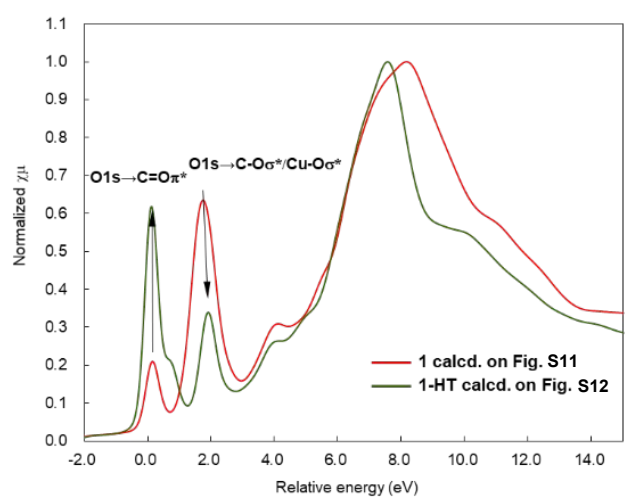

Figure S25. DFT-calculated O K-edge NEXAFS spectra of $\mathbf{1}$ and 1HT. Gaussian peak smearing based on an instrumental broadening $0.18 \mathrm{eV}$ and a life-time effect corresponding to core-hole condition $0.1 \mathrm{eV}$ are performed. 

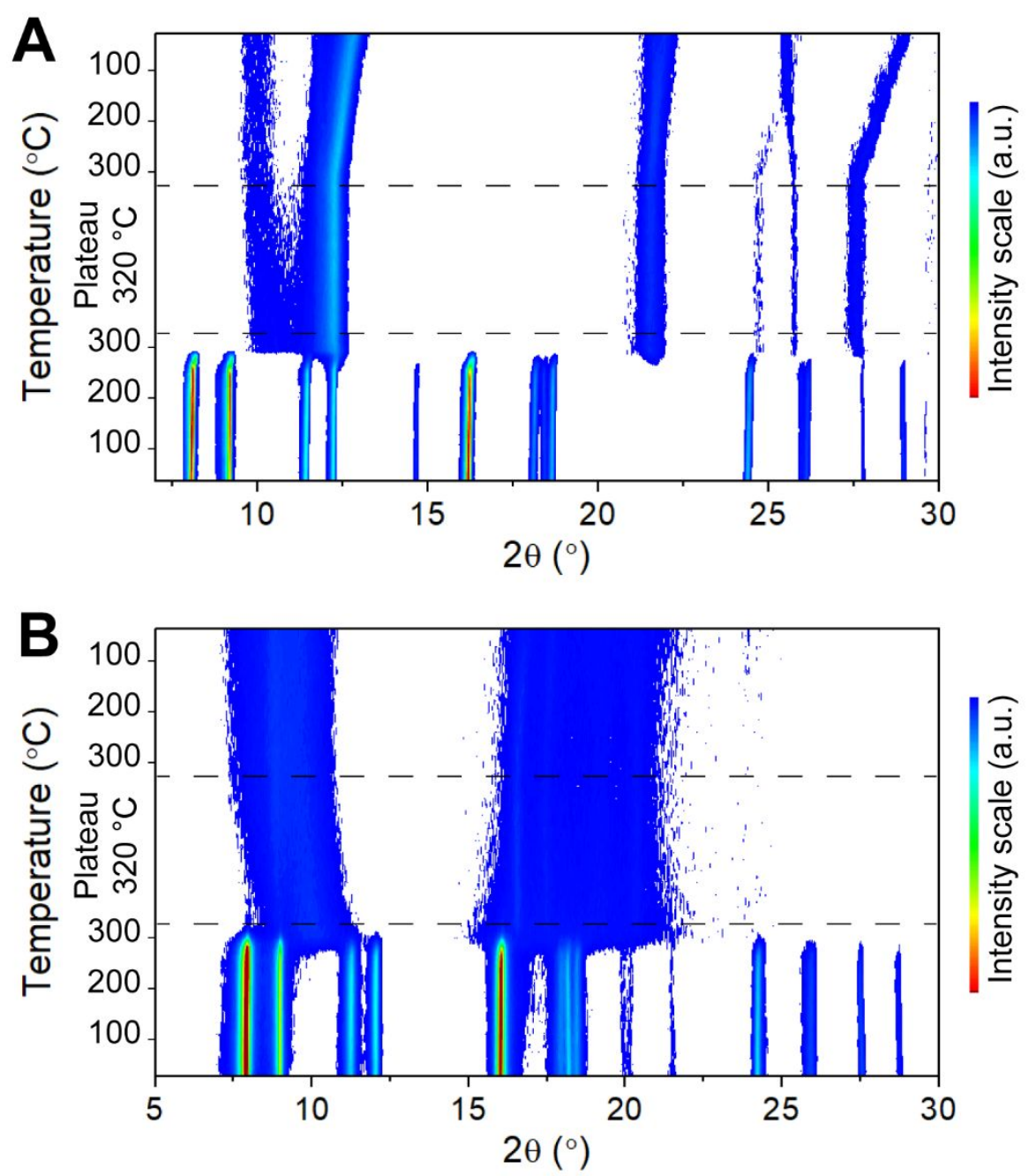

Figure S26. Variable temperature PXRD during one heat-treatment cycle (A: 1; B: 1つPSt). Heating and cooling rates: $3^{\circ} \mathrm{C} / \mathrm{min}$. Samples were maintained $2 \mathrm{~h}$ at $320^{\circ} \mathrm{C}$. This measurement confirms the loss of structure of the host MOF 1 around $300{ }^{\circ} \mathrm{C}$ when DABCO was removed, as indicated by the disappearance of the corresponding diffraction peaks. At the same time, phases analogue of 1HT and 1HTつPSt were formed, with a slightly different pattern due to the high temperature. Upon cooling, the diffraction peaks of the high temperature analogues were maintained, but shifted progressively to reach the patterns of the room temperature phases. In addition, no new crystalline phase was formed during the cooling. 


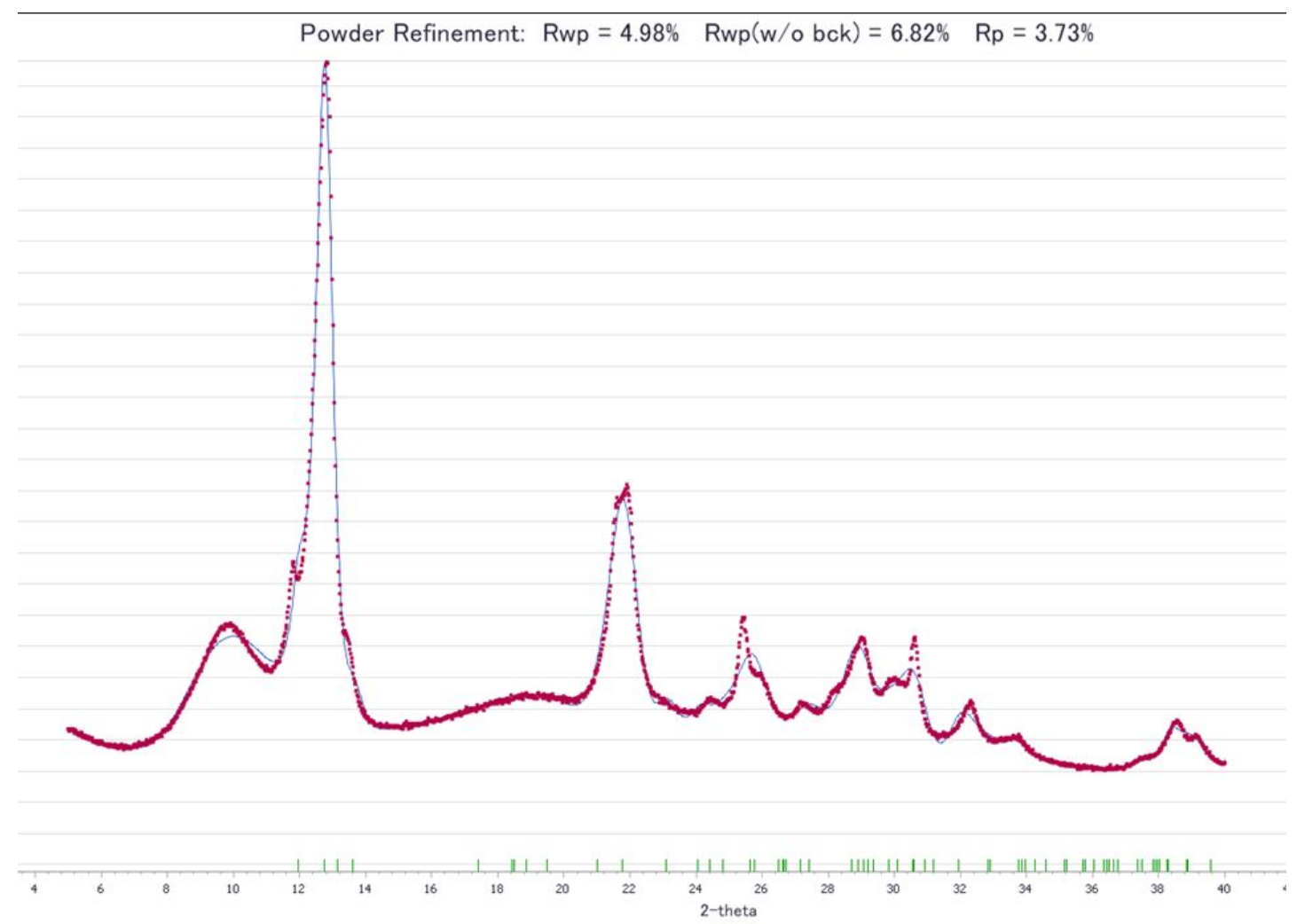

Figure S27. Pawley refinement for the PXRD pattern $(\mathrm{Cu}, \lambda=0.154 \mathrm{~nm})$ of $\mathbf{1 H T}$. The pattern of the turbostratic phase impurity was broad enough and of low enough intensity to be considered as a background for the measurement. $\mathrm{a}=8.234 \AA ; \mathrm{b}=7.709 \AA ; \mathrm{c}=6.525 \AA ; \alpha=88.948^{\circ} ; \beta=$ $94.658^{\circ} ; \gamma=115.729^{\circ} ; \mathrm{V}=371.81 \AA^{3} ; \mathrm{R}_{\mathrm{p}}=3.73 \%$.

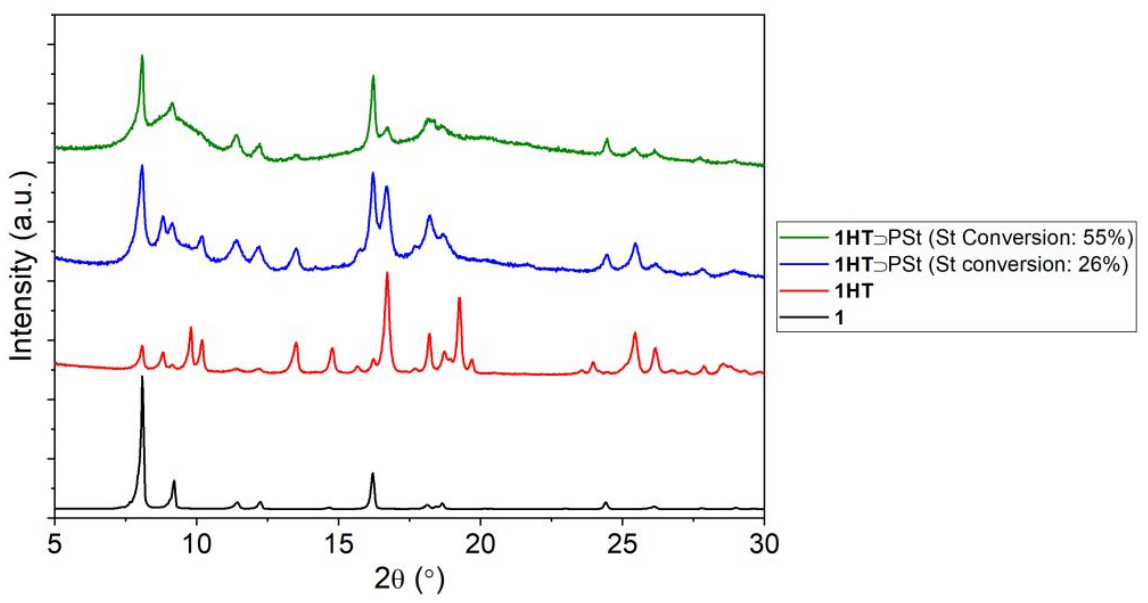

Figure S28. Reinsertion of DABCO in 1HT and 1HT $\supset$ PSt. Depillared samples were placed in a solution of DABCO in nitromethane $(25 \mathrm{mg} / \mathrm{ml})$, and let to react for $16 \mathrm{~h}$ at room temperature. Nitromethane was chosen as solvent because it did not degrade 1HT and did not dissolve PSt. 

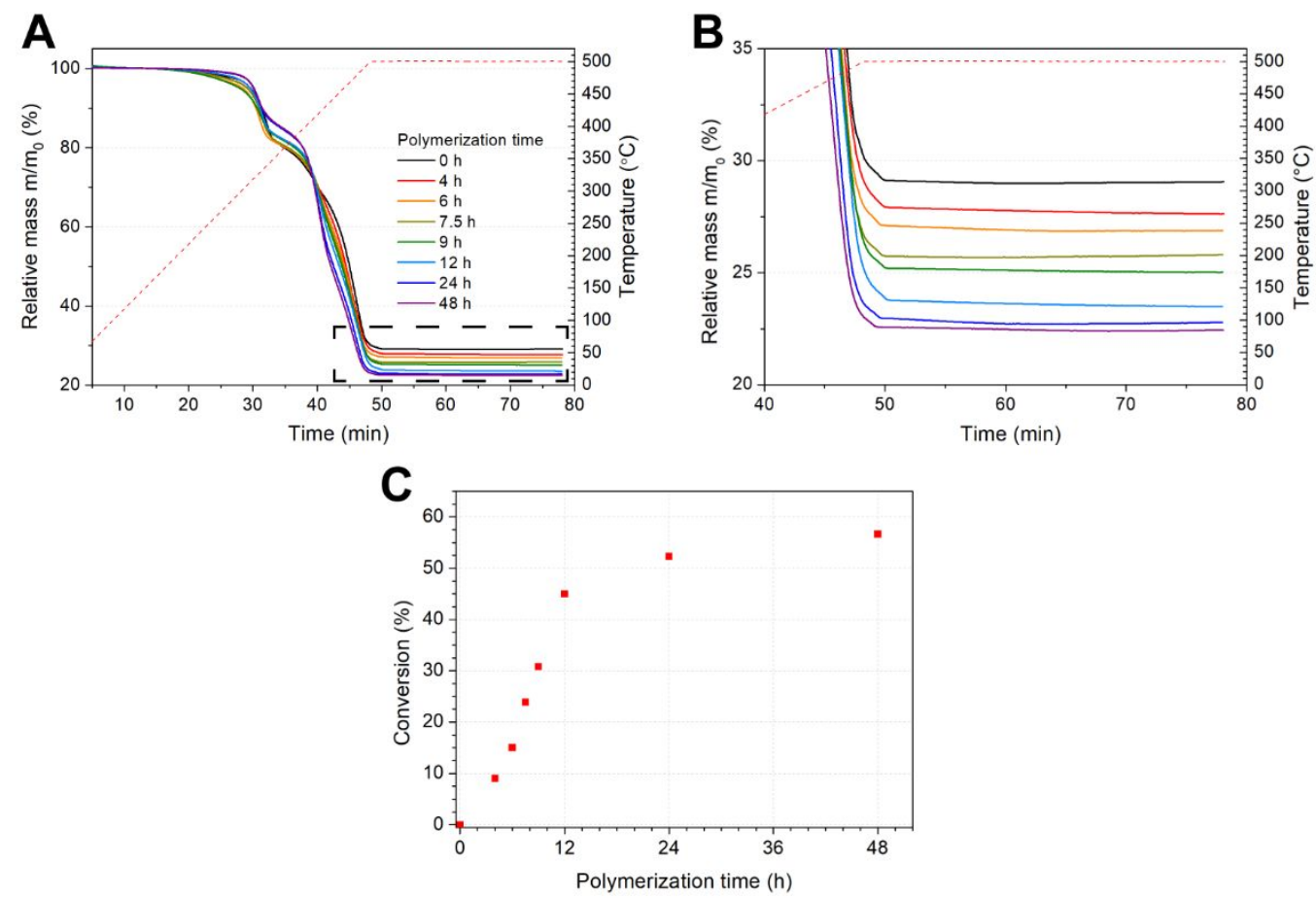

Figure S29. Determination of PSt loading in 1دPSt composites as a function of the polymerization time, using thermogravimetric analysis (TGA) under air (A, B). Dotted line: temperature. The reference mass $\mathrm{m}_{0}$ was determined at $150{ }^{\circ} \mathrm{C}$, after desorption of physisorbed water. For all polymerization times, the TGA exhibited the same decomposition steps (A). At 500 ${ }^{\circ} \mathrm{C}$, a plateau was reached, with a residual mass $r(\mathbf{B})$. (C) Plot of the conversion as a function of the polymerization time. Conversion was determined as follows. Considering that PSt was completely decomposed at this temperature $\left(r_{P S t}=0\right)$, and that 1 was converted into $\mathrm{ZnO}$ with $r_{M O F}=0.29$, the mass fraction of PSt in the composite $x$ was determined with the equation $r=r_{P S t}$ $x+r_{M O F}(1-x)$. The PSt loading $L$ (i.e. the mass of PSt compared to the mass of empty MOF) was deduced using the relationship $L=x /(1-x)$. The conversion was determined considering the initial loading of St $\left(L_{S t}=52 \%\right)$. 


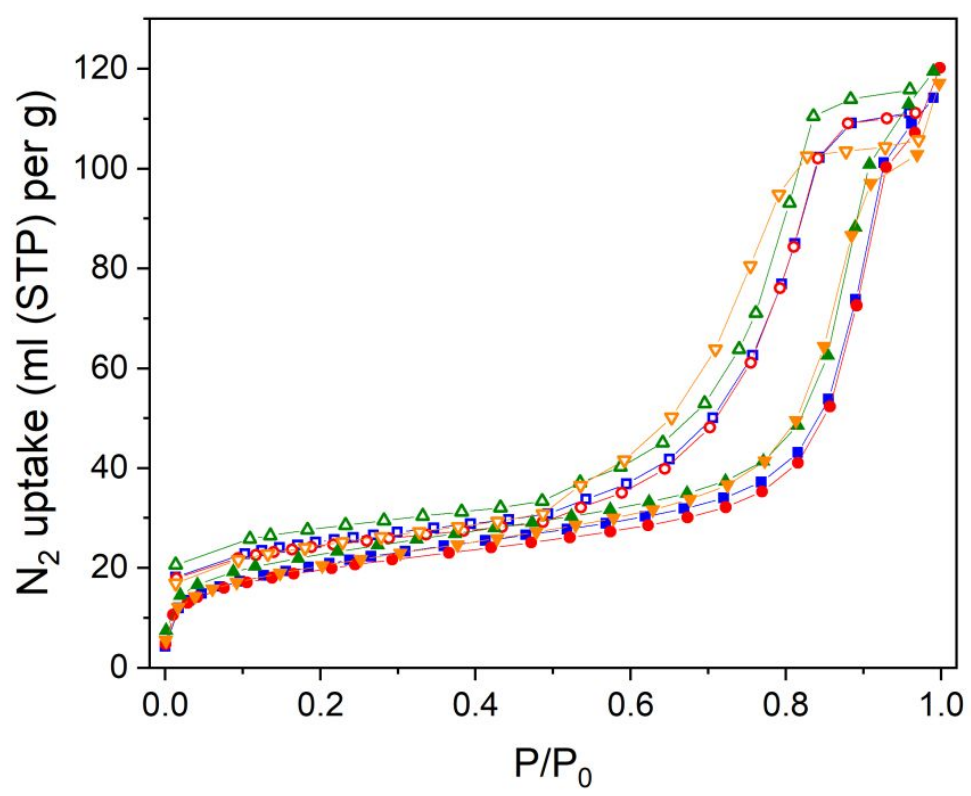

Figure S30. Evaluation of the reproducibility of the depillaring treatment. $\mathrm{N}_{2}$ adsorption isotherms $(77 \mathrm{~K})$ are presented for several samples prepared in similar conditions. The adsorption isotherms are all very similar, confirming the reproducibility of the sample preparation and the reversibility of the $\mathrm{N}_{2}$ adsorption. (i) Blue squares: 1HTدPSt (Polymerization time: $7.5 \mathrm{~h}$; St conversion: 26\%). (ii) Red circles: Same sample as (i), measured a second time. (iii) Green upward triangles: Same batch of $1 \supset$ PSt, different instance of heat-treatment. (iv) Orange downward triangles: Different batch of 1, different instances of PSt formation and heat-treatment. 


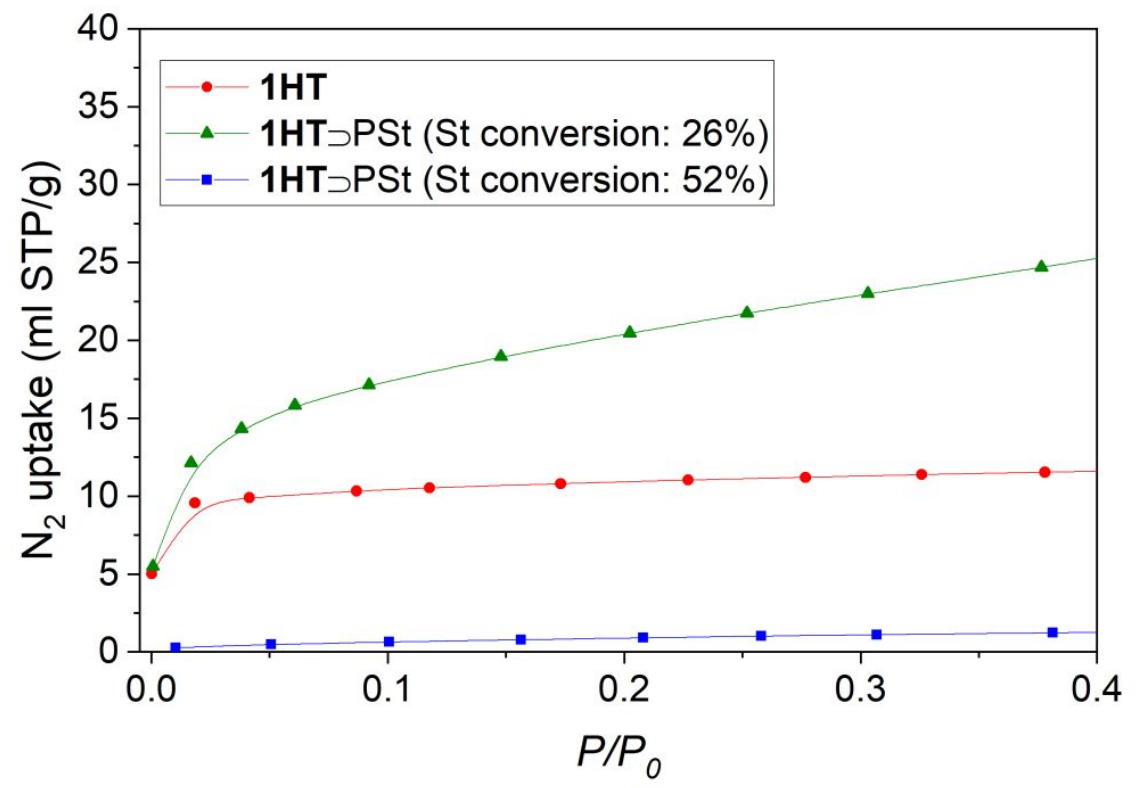

Figure S31. Close-up view on the low-pressure domain for the adsorption of $\mathrm{N}_{2}$ at $77 \mathrm{~K}$. The

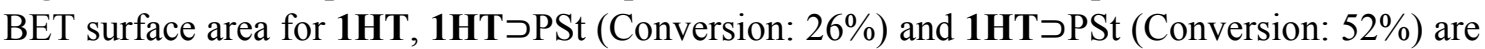
$40 \mathrm{~m}^{2} / \mathrm{g}, 72 \mathrm{~m}^{2} / \mathrm{g}, 3 \mathrm{~m}^{2} / \mathrm{g}$ respectively. However, one should note that the BET formalism does not consider the possibility of a dynamic behavior for the adsorbent. While this is suitable for rigid MOFs with a permanent microporosity (such as $\mathbf{1}$ and $\mathbf{1} \supset \mathrm{PSt}$ ), it has little relevance for dynamic materials. This is especially true for materials with a gate-opening, where the accessible volume (and thus, the BET surface area) in the closed phase are low. Instead, it is recommended to consider the complete adsorption profiles for these materials, and notably the pressures of gateopening, as well as the maximum guest capacities in the desired range. 

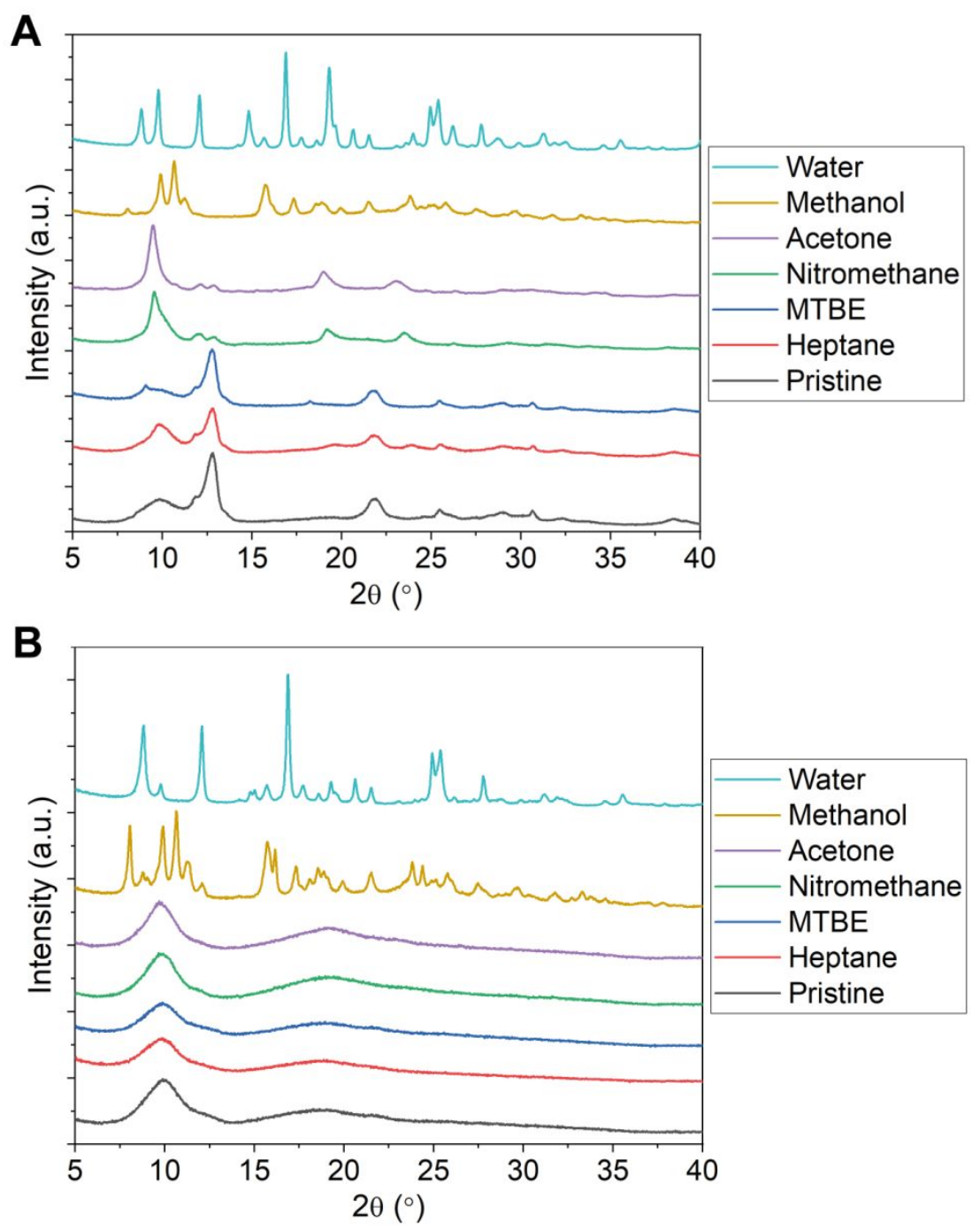

Figure S32. PXRD of 1HT (A) and 1HTدPSt (St conversion: 26\%, B) in several solvents. In protic solvents such as water and methanol, both materials were unstable and new crystalline phases were formed. This was likely caused by a reaction at the open metal sites. In the case of 1HT, other solvents caused either limited changes (e.g. heptane, MTBE (methyl-tert-butyl ether)), or a reversible peak shift toward lower angles (e.g. nitromethane and acetone). By contrast, 1HT $\supset$ PSt maintained similar PXRD patterns in all these solvents. As such, the adsorption in 1HT $\supset$ PSt was not associated to local changes of structure visible by PXRD. 
A

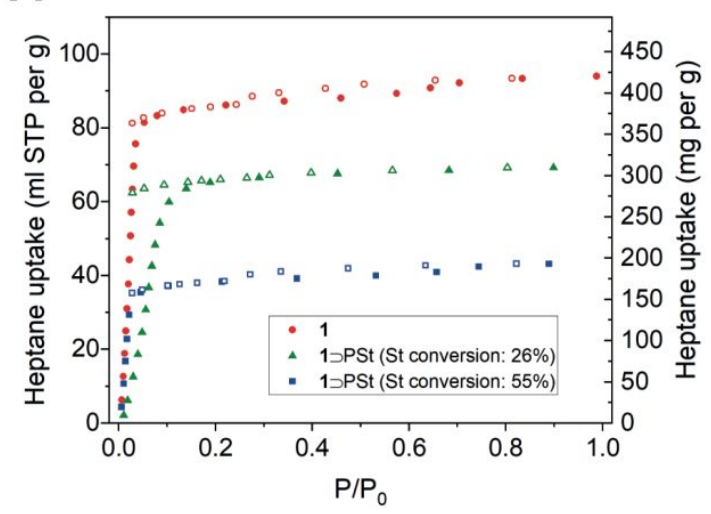

B

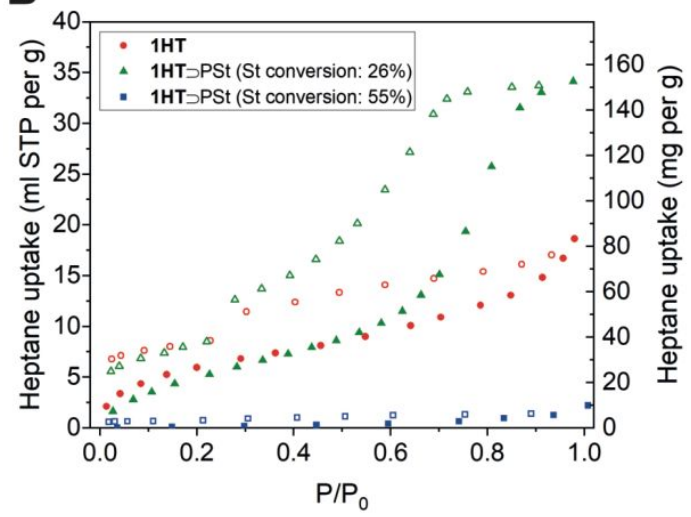

Figure S33. $n$-Heptane adsorption isotherms (measured at $293 \mathrm{~K}$ ) for the pillared materials 1 and 1HT $\supset$ PSt (A), and the heat-treated 1HT and 1HTつPSt (B). Several PSt conversions are presented. 


\section{References}

1. Dybtsev, D. N.; Chun, H.; Kim, K., Rigid and Flexible: A Highly Porous Metal-Organic Framework with Unusual Guest-Dependent Dynamic Behavior. Angew. Chem. Int. Ed. 2004, 43 (38), 5033-5036.

2. The fitting calculation was performed by the FEFF6 program (revision 9.6.4) embedded with Artemis. For FEFF6 program, see: a) Rehr, J. J.; Kas, J. J.; Vila, F. D.; Prange, M. P.; Jorissen, K., Parameter-free calculations of X-Ray spectra with FEFF9. Phys. Chem. Chem. Phys. 2010, 12, 5503-5513. b) Rehr, J. J.; Albers, R. C., Theoretical approaches to x-ray absorption fine structure. Rev. Mod. Phys. 2000, 72, 621-654. For Artemis software, see: c) Ravel, B.; Newville, M., ATHENA, ARTEMIS, HEPHAESTUS: data analysis for X-ray absorption spectroscopy using IFEFFIT. J. Synchrotron Rad. 2005, 12, 537-541.

3. Ohashi, H.; Ishiguro, E.; Tamenori, Y.; Kishimoto, H.; Tanaka, M.; Irie, M.; Tanaka, T.; Ishikawa, T., Outline of soft X-ray photochemistry beamline BL27SU of SPring-8. Nucl. Instrum. Methods Phys. Res. A 2001, 467, 529-532.

4. Hatsui, T.; Shigemasa, E.; Kosugi, N., Design of a transmission grating spectrometer and an undulator beamline for soft $\mathrm{x}$-ray emission studies. AIP Conf. Proc. 2004, 705 (1), 921-924.

5. Clark, S. J.; Segall, M. D.; Pickard, C. J.; Hasnip, P. J.; Probert, M. J.; Refson, K.; Payne, M. C., First principles methods using CASTEP. Zeitschrift Fur Kristallographie 2005, 220 (5-6), 567-570.

6. Mizoguchi, T.; Tanaka, I.; Gao, S.-P.; Pickard, C. J., First-principles calculation of spectral features, chemical shift and absolute threshold of ELNES and XANES using a plane wave pseudopotential method. J. Phys.: Condens. Matter 2009, 21 (10), 104204.

7. Distefano, G.; Suzuki, H.; Tsujimoto, M.; Isoda, S.; Bracco, S.; Comotti, A.; Sozzani, P.; Uemura, T.; Kitagawa, S., Highly ordered alignment of a vinyl polymer by host-guest crosspolymerization. Nat. Chem. 2013, 5 (4), 335-341.

8. Uemura, T.; Yanai, N.; Watanabe, S.; Tanaka, H.; Numaguchi, R.; Miyahara, M. T.; Ohta, Y.; Nagaoka, M.; Kitagawa, S., Unveiling thermal transitions of polymers in subnanometre pores. Nat. Commun. 2010, 1, 83. 